\title{
Derivation of stabilized equations for numerical solution of advective-diffusive transport and fluid flow problems
}

\author{
Eugenio Oñate \\ International Center for Numerical Methods in Engineering, Universidad Politécnica de Cataluña, Gran Capitán s/n. 08034 Barcelona. \\ Spain
}

Dedicated to J. Tinsley Oden on the occasion of his 60th birthday

\begin{abstract}
The concept of the so-called 'artificial or balancing diffusion' used to stabilize the numerical solution of advective-diffusive transport and fluid flow problems is revised in this paper. It is shown that the standard forms of the balancing diffusion terms, usually chosen in a heuristic manner, can be naturally found by introducing higher-order approximations in the derivation of the governing differential equations via standard conservation (or equilibrium) principles. This allows us to reinterpret many stabilization algorithms and concepts used in every-day practice by numerical analysts and also provides an expression for computing the stabilization parameter.
\end{abstract}

\section{Introduction}

It is widely accepted that the accurate numerical solution of advective-diffusive transport and fluid flow problems using either finite difference (FD) [1], finite volume (FV) [1,2], finite element (FE) [3-12], or finite point (FP) methods $[13,14]$ requires invariably the addition of some balancing diffusion (also called 'artificial diffusion') terms. The role of this apparently non-physical diffusion is typically two fold: (a) to counter-balance the underdiffusive character of most integration schemes in space (such as the central difference scheme in FD or FV methods, or the equivalent Galerkin form in FEM, etc.), and (b) to stabilize the numerical solution in the vicinity of high gradients (such as wave shocks, etc.) by smoothing out local oscillations.

Although extensive work has been devoted to the development of stabilization procedures in FD, FV and FE methods, it is acknowledged that most of the existing procedures are based on somewhat heuristic arguments. Indeed, it is widely accepted that the origins and precise definition of the stabilization parameters used in numerical computations are, in most cases, unsolved mysteries whose solution has motivated much research in recent years mainly within the FEM context [14,16-18].

The purpose of this paper is to show that the stabilization terms emerge naturally in the governing differential equations of the problem, once the concept of flow balance (or equilibrium) over a 'finite' domain is accepted. This allows us to reinterpret the stabilization terms as an intrinsic and natural contribution to the original differential equations, instead of a correction term introduced at the discretization level as it is usually understood by most FD, FV and FE practitioners. Moreover, by exploiting this natural stabilization concept, the standard forms of the balancing terms appearing in many well-known stabilized schemes for advective-diffusive and fluid flow problems such as direct artificial diffusion, upwinding and Petrov-Galerkin weighting, Streamline Upwind Petrov-Galerkin (SUPG), Subgrid Scale (SS), Galerkin Least Squares (GLS), Lax-Wendroff, Characteristic Galerkin, Laplacian pressure operator etc. are easily recognized and can be reinterpreted in a more physical manner. 
An additional important benefit from the approach proposed is that it provides an expression for computing numerically the stabilization parameter.

The layout of the paper is the following. In the next section the stabilization procedure is detailed for one-dimensional advective-diffusive problems. The two-dimensional case is considered next. The stabilization method is then generalized to the case of compressible and incompressible flows. Analogies of the proposed methodology with standard stabilization procedures are presented in all cases. The generalization of the method to incorporate higher-order stabilization terms is also shown. The paper finishes with a proposal for computing numerically the stabilization parameter. The accuracy of the method is shown in the study of a simple advective-diffusive problem.

\section{The one-dimensional advective-diffusive problem}

\subsection{Basic governing equations}

Let us consider for simplicity, the standard convection-diffusion transport problem to be solved in a one-dimensional domain of length $l$ (Fig. 1(a)). Fig. 1(b) shows a typical segment $A B$ of length $\overline{A B}=h$ where balance (equilibrium) of fluxes must be satisfied. The values of the diffusive flow rate $q$ and the advective transport rate $u \phi$ at a point $A$ with coordinate $x_{A}=x_{B}-h$ can be approximated in terms of values at point $B$ using a second-order Taylor's expansion in the standard manner as

$q_{A}=q\left(x_{B}-h\right)=q\left(x_{B}\right)-\left.h \frac{\mathrm{d} q}{\mathrm{~d} x}\right|_{B}+\mathrm{O}\left(h^{2}\right)$
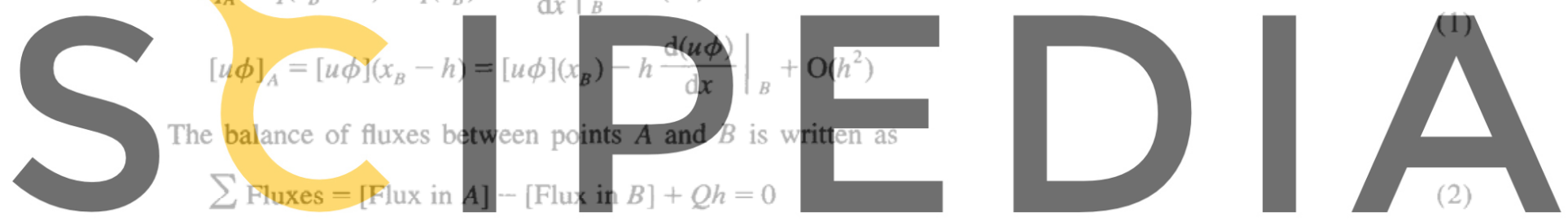

or

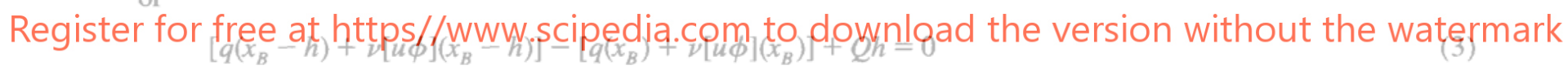

In Eqs. (1)-(3) $\phi$ is the unknown transported variable, $u$ is the known velocity field which is taken as positive if it acts in the direction of the $x$ axis, $\nu$ is the advective material parameter which is assumed to be constant and $Q$ is a distributed source which is assumed here to act uniformly over the domain $A B$.

Substituting Eqs. (1) into Eq. (3) and noting that the position of point $B$ is arbitrary, i.e. $x_{B}=x$, gives after simplification

(b)

(a)

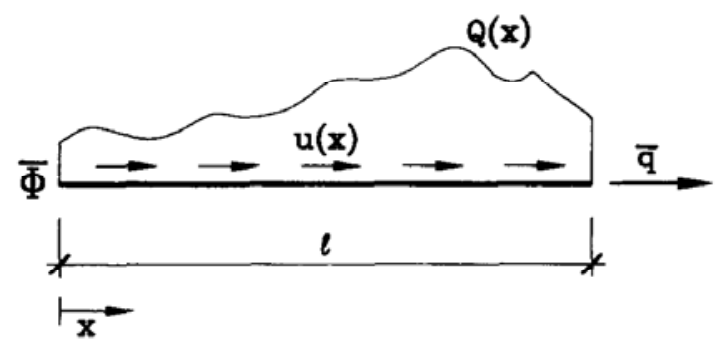

Q

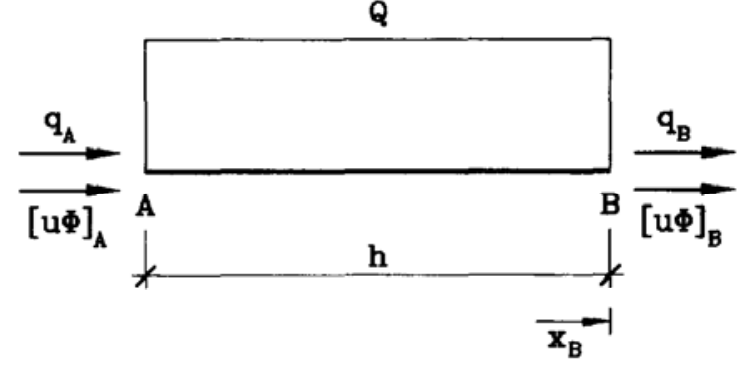

$$
\begin{gathered}
q_{B}=q\left(x_{B}\right) ; q_{A}=q\left(x_{A}\right)=q\left(x_{B}-h\right) \\
\left.[u \Phi]_{B}=[u \Phi]\right]\left(x_{B}\right) ;[u \Phi]_{A}=[u \Phi]\left(x_{A}\right)=[u \Phi]\left(x_{B}-h\right)
\end{gathered}
$$

Fig. 1. (a) One-dimensional convection-diffusion problem; (b) finite balance domain $A B$. 


$$
-\nu \frac{\mathrm{d}[u \phi]}{\mathrm{d} x}-\frac{\mathrm{d} q}{\mathrm{~d} x}+Q=0
$$

The diffusive flow rate $q$ is related to the change of the unknown variable $\phi$ by Fourier's law as $q=-k \mathrm{~d} \phi / \mathrm{d} x$. Substituting this equation into Eq. (4) leads to the standard form of the advection-diffusion equation

$$
-\nu \frac{\mathrm{d}[u \phi]}{\mathrm{d} x}+\frac{\mathrm{d}}{\mathrm{d} x}\left[k \frac{\mathrm{d} \phi}{\mathrm{d} x}\right]+Q=0
$$

Clearly, if $u$ is constant this gives

$$
-\nu u \frac{\mathrm{d} \phi}{\mathrm{d} x}+\frac{\mathrm{d}}{\mathrm{d} x}\left[k \frac{\mathrm{d} \phi}{\mathrm{d} x}\right]+Q=0
$$

The transient case is obtained by invoking again the conservation law, i.e.

$$
\sum \text { Fluxes }=\nu \frac{\partial}{\partial t} \int_{0}^{h} \phi d x
$$

It is usual to assume that $\phi$ is constant within the balancing domain for computation of the integral in the r.h.s. of Eq. (7a), i.e.

$$
\frac{\partial}{\partial t} \int_{0}^{h} \phi \mathrm{d} x=h \frac{\partial \phi}{\partial t}
$$

The same expression can be obtained by using the

he r.h.s. of Eq. (7b). A higher ordqr

(2) - (7) the standard transien

$\nu\left(\frac{\partial \phi}{\partial t}+\frac{\partial[u \phi]}{\partial x}\right)$
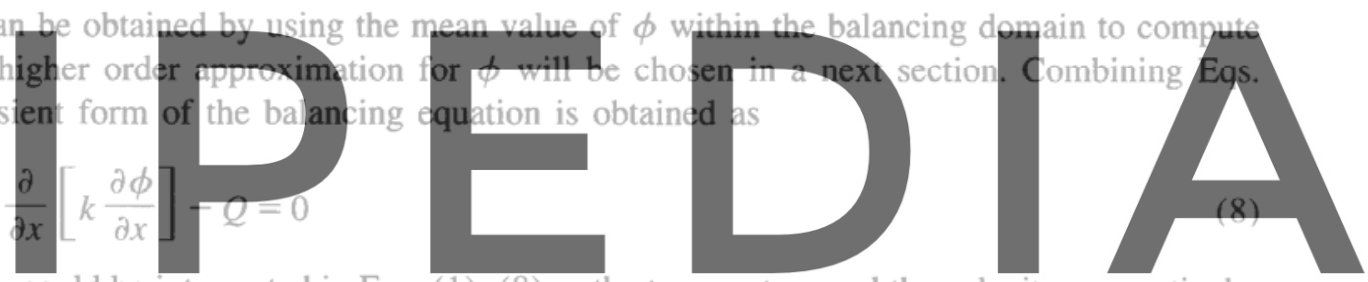

As an example, $\phi$ and $u$ could be interpreted in Eqs. (1)-(8) as the temperature and the velocity, respectively,

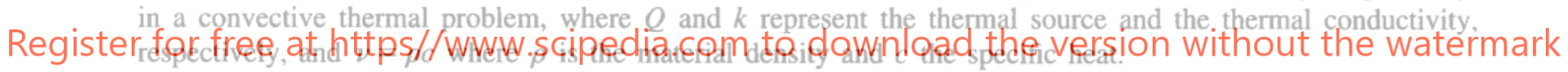

The problem is completed with the following spatial and temporal boundary conditions.

Prescribed value of $\phi$ (Dirichlet b.c.)

$$
\phi-\bar{\phi}=0 \quad \text { on } x=0
$$

where $\bar{\phi}$ is the prescribed unknown field at the Dirichlet boundary.

Prescribed total flux (Neumann b.c.)

$$
q,-\bar{q}=0 \quad \text { on } x=l
$$

where $\bar{q}$ is the prescribed total flux at the Neumann boundary. The total flux $q_{t}$ can be splitted into the advective and diffusive contributions as

$$
q_{t}=\nu u \phi+q=\nu u \phi-k \frac{\mathrm{d} \phi}{\mathrm{d} x}
$$

Substituting (11) into (10) gives

$$
-\nu u \phi+k \frac{\mathrm{d} \phi}{\mathrm{d} x}+\bar{q}=0 \quad \text { on } x=l
$$

Initial condition

$$
\phi-\phi_{0}=0 \text { for } t=t_{0}
$$


where $\phi_{0}$ is the value of $\phi$ at some initial time $t_{0}$.

REMARK 1 . In many occasions only the diffusive flux is prescribed at the Neumann boundary. Eq. (12) is simply written in these cases as

$$
k \frac{\mathrm{d} \phi}{\mathrm{d} x}+\bar{q}=0 \quad \text { on } x=l
$$

\subsection{The concept of balancing diffusion}

Let us assume now that the advective transport term has an important variation over the segment $A B$. In order to capture this variation, the advective term will be now expanded in Taylor series up to third order as

$$
[u \phi]\left(x_{B}-h\right)=[u \phi]\left(x_{B}\right)-\left.h \frac{\mathrm{d}[u \phi]}{\mathrm{d} x}\right|_{B}+\left.\frac{h^{2}}{2} \frac{\mathrm{d}^{2}[u \phi]}{\mathrm{d} x^{2}}\right|_{B}-\mathrm{O}\left(h^{3}\right)
$$

Substituting this equation into Eq. (3) gives

$$
-\nu \frac{\mathrm{d}[u \phi]}{\mathrm{d} x}+\frac{h}{2} \frac{\mathrm{d}^{2}[u \phi]}{\mathrm{d} x^{2}}-\frac{\mathrm{d} q}{\mathrm{~d} x}+Q=0
$$

and using Fourier's law yields finally

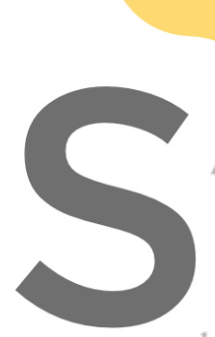

$-v \frac{\mathrm{d}[u \phi]}{\mathrm{d} x}+\frac{h}{2} \frac{\mathrm{d}^{2}[u \phi]}{\mathrm{d} x^{2}}+\frac{\mathrm{d}}{\mathrm{d} x}\left(k \frac{\mathrm{d} \phi}{\mathrm{d} x}\right)+Q=0$

Assuming now $u$ to be constant, Eq. (17) gan be rowritten as
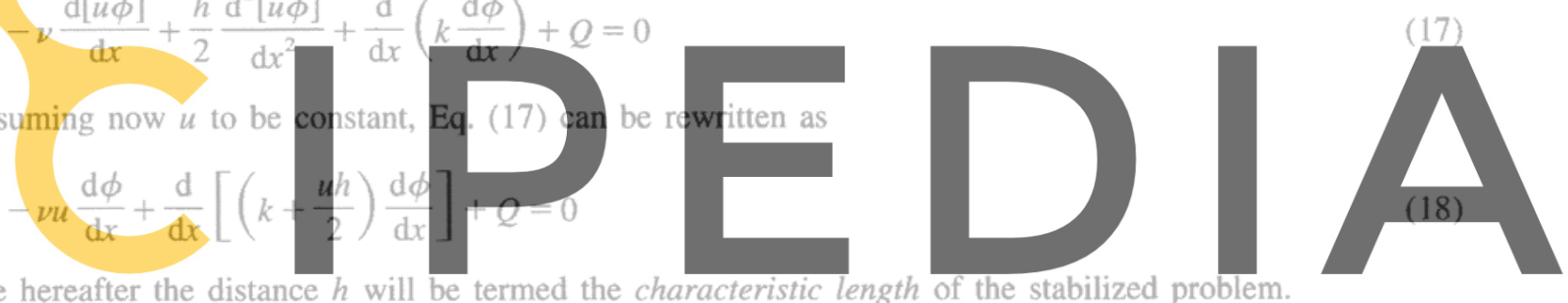

where hereafter the distance $h$ will be termed the characteristic length of the stabilized problem.
Note that the effect of accounting for a higher approximation of the advective term introduces 'naturally' an

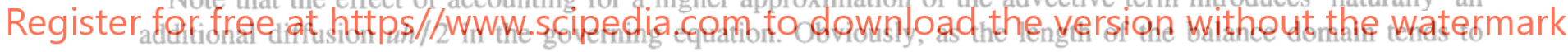

zero the standard 'infinitesimal' form of the advection-diffusion equation is recovered. The problem remains

now to find the value of the characteristic length $h$ so as to ensure accuracy and stability of the numerical solution of Eq. (18).

The 'stabilized' transient form equivalent to Eq. (8) can be now written as

$$
\nu\left(\frac{\partial \phi}{\partial t}+\frac{\partial(u \phi)}{\partial x}\right)-\frac{\partial}{\partial x}\left(k \frac{\partial \phi}{\partial x}\right)-\frac{h}{2} \frac{\partial^{2}(u \phi)}{\partial x^{2}}-Q=0
$$

or

$$
\nu\left(\frac{\partial \phi}{\partial t}+u \frac{\partial \phi}{\partial x}\right)-\frac{\partial}{\partial x}\left[\left(k+\frac{u h}{2}\right) \frac{\partial \phi}{\partial x}\right]-Q=0
$$

for a constant velocity $u$.

REMARK 2. The characteristic length $h$ can be written as $h=2 \tau u$ where $\tau$ is the so-called intrinsic time scale in the convective transport and fluid flow stabilization literature [5]. Note that $\tau$ is the time for a particle to travel the distance $h / 2$ at a speed equal to $u$.

REMARK 3. The characteristic length $h$ is usually defined in $\mathrm{FE}$ and $\mathrm{FV}$ procedures as $\alpha l^{(e)}$ where $\alpha$ is a stabilization (upwinding) parameter and $l^{(e)}$ is a characteristic element dimension (i.e. the element length for 1D problems). The search for the optimal characteristic length $l$ or the optimum intrinsic time scale $\tau$ is, therefore, equivalent to finding the optimum value of the stabilization parameter $\alpha$. This topic is discussed in Section 8 . 


\subsection{Generalization of the stabilization process}

Let us assume now that the changes in the advective and diffusive terms over the balance segment are so important that a higher-order expansion is required for all terms. The balance equation (3) reads now

$$
q(x)+\nu[u \phi](x)-q(x-h)-\nu[u \phi](x-h)-\frac{1}{2}[Q(x)+Q(x-h)] h=0
$$

where a linear distribution of the external source $Q$ over the balance domain $A B$ has been assumed (see Fig. 2). Note that in Eq. (15) $x_{B}=x$ has been taken.

The following expansions can be written

$$
\begin{aligned}
& q(x-h)=q(x)-h \frac{\mathrm{d} q}{\mathrm{~d} x}+\frac{h^{2}}{2} \frac{\mathrm{d}^{2} q}{\mathrm{~d} x^{2}}+\mathrm{O}\left(h^{3}\right) \\
& Q(x-h)=Q(x)-h \frac{\mathrm{d} Q}{\mathrm{~d} x}+\mathrm{O}\left(h^{2}\right)
\end{aligned}
$$

\section{Substituting Eqs. (15) and (21) into (20) gives after simplification}

$$
\nu \frac{\mathrm{d}(u \phi)}{\mathrm{d} x}+\frac{\mathrm{d}}{\mathrm{d} x}\left(k \frac{\mathrm{d} \phi}{\mathrm{d} x}\right)+Q-\frac{h}{2} \frac{\mathrm{d}}{\mathrm{d} x}\left[-\nu \frac{\mathrm{d}(u \phi)}{\mathrm{d} x}+\frac{\mathrm{d}}{\mathrm{d} x}\left(k \frac{\mathrm{d} \phi}{\mathrm{d} x}\right)+Q\right]=0
$$

Eq. (22) can be rewritten a more compact form as
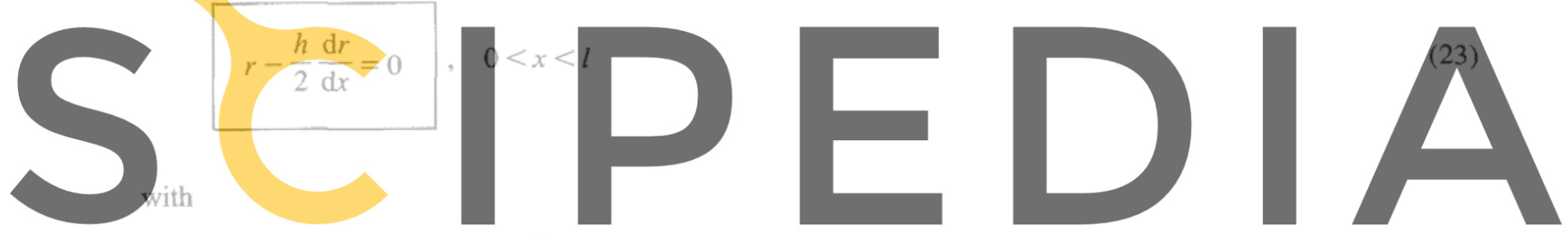

$\frac{\mathrm{d}(u \phi)}{2 \mathrm{~d} x}+\frac{\mathrm{d}}{\operatorname{de}}\left(k \frac{\mathrm{d} \phi}{\mathrm{d} x}\right)+Q$

(24)

Register for free at Https/fwww.Scipedia.com to download the version without the watermark

\subsection{Stabilized Neumann boundary condition}

The essential (Dirichlet) boundary condition to Eq. (23) is the standard one given by Eq. (9).

To obtain the stabilized Neumann boundary condition we write the balance equation at a boundary point.

For reasons which will be evident later, the length of the balance segment $A B$ at the boundary is taken now as half of the characteristic length $h$ for the interior domain. The balance equation is now, assuming now the source $Q$ to be constant over $A B$ (see Fig. 3).
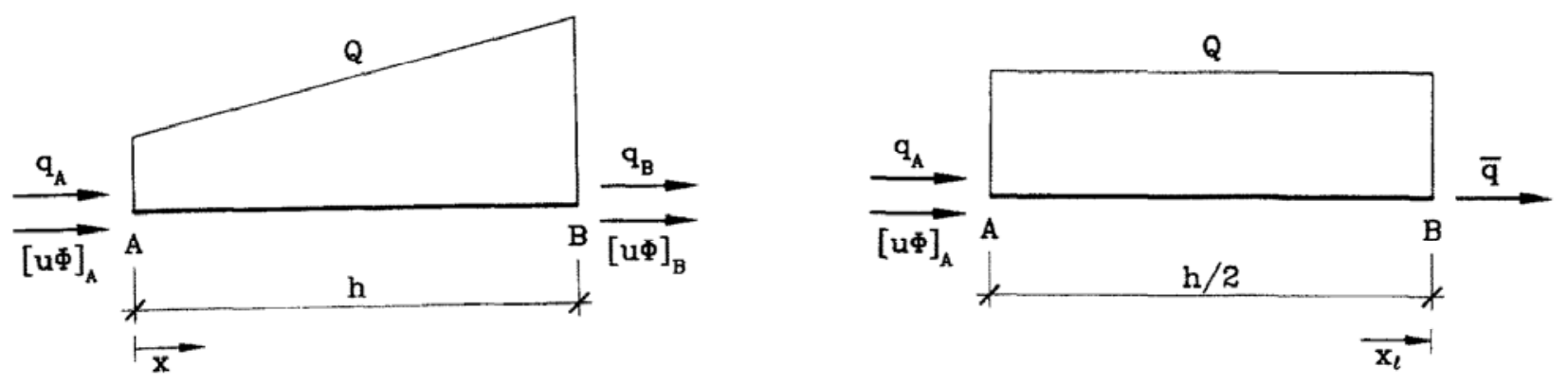

Fig. 2. Equilibrium of fluxes in a finite balance domain with linear external source.

Fig. 3. Balance domain next to a Neumann boundary point $B$. 


$$
\bar{q}-q\left(x_{A}\right)-\nu[u \phi]_{\left(x_{a}\right)}-\frac{h}{2} Q=0
$$

where $\bar{q}$ is the prescribed total flux at $x=l$ and $x_{A}=x_{B}-h / 2$.

Let us now express the advective and diffusive fluxes at point $A$ in terms of second-order expansions via Eqs. (1). After little algebra we obtain

$$
-\nu u \phi+k \frac{\mathrm{d} \phi}{\mathrm{d} x}+\bar{q}-\frac{h}{2} r=0 \quad \text { on } x=l
$$

where $r$ is given by Eq. (24).

Eq. (23) can now be solved together with Eqs. (9) and (26). These equations are the starting point to derive stabilized numerical schemes using FD, FE, FV and FP methods. The importance of using the stabilized form (26) is discussed in [14] in the context of the FP method.

REMARK 4. In the case that only the diffusive flux is prescribed at the Neumann boundary equation (26) is written as

$$
k \frac{\mathrm{d} \phi}{\mathrm{d} x}+\bar{q}-\frac{h}{2} r=0 \quad \text { on } x=l
$$
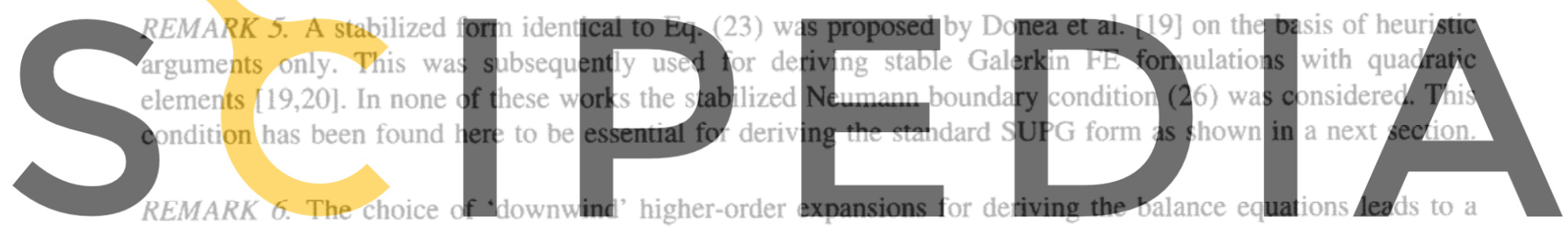

change of the negative sign in the stabilization term in Eqs. (23) and (26) to a positive one. However, a study of

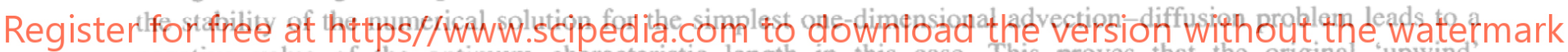
negative value of the optimum characteristic length in this case. This proves that the original "upwind" expansions chosen here are the correct ones leading to positive values of $h$ in all cases.

REMARK 7. The analytical solution of Eq. (23) is

$$
r=A e^{(2 x) / h}
$$

Using Eq. (26) gives

$$
A=\frac{2}{h} e^{h /(2 L)}\left[-\nu u \phi+k \frac{\mathrm{d} \phi}{\mathrm{d} x}+\bar{q}\right]_{x=l}
$$

The solution is therefore written as

$$
r=\frac{2}{h} e^{h /(2 L)}\left[-\nu u \phi+k \frac{\mathrm{d} \phi}{\mathrm{d} x}+\bar{q}\right]_{x=l} e^{(2 x) / h}
$$

Note that exact pointwise satisfaction of the Neumann boundary condition at $x=l$, i.e. $-\nu u \phi+k \mathrm{~d} \phi / \mathrm{d} x+$ $\bar{q}=0$, gives $r=0$ in the whole analysis domain and the equivalent problem can be written in the standard form

$$
\begin{array}{ll}
r=0,0<x<l & \\
\phi-\bar{\phi}=0 & \text { on } x=0 \\
-\nu u \phi+k \frac{\mathrm{d} \phi}{\mathrm{d} x}+\bar{q}=0 & \text { on } x=l
\end{array}
$$


Eq. (30) therefore expresses the effect in the global solution of the error in satisfying the Neumann boundary condition.

\subsection{General stabilized transient form}

The stable form of the transient balance equation is obtained by substituting Eq. (22) into (7a). For consistency with previous section let us assume now a linear variation of $\phi$ within the balancing domain giving

$$
\frac{\partial}{\partial t} \int_{0}^{h} \phi(x) \mathrm{d} x=\frac{\partial}{\partial t}\left(\frac{\phi_{A}+\phi_{B}}{2}\right) h=\frac{\partial}{\partial t}\left(\phi-\frac{h}{2} \frac{\partial \phi}{\partial x}\right) h
$$

In the derivation of Eq. (32) use of the first-order approximation $\phi_{A}=\phi_{B}-h \partial \phi /\left.\partial x\right|_{B}$ has been made. Also, as usual $x_{B}=x$ has been taken. Combining Eqs. (7a), (21) and (32) gives after simplification

$$
\nu \frac{\partial \phi}{\partial t}-r-\frac{h}{2} \frac{\partial}{\partial x}\left(\nu \frac{\partial \phi}{\partial t}-r\right)=0
$$

It is interesting to note that Eq. (33) can be casted in the same form (23), i.e.
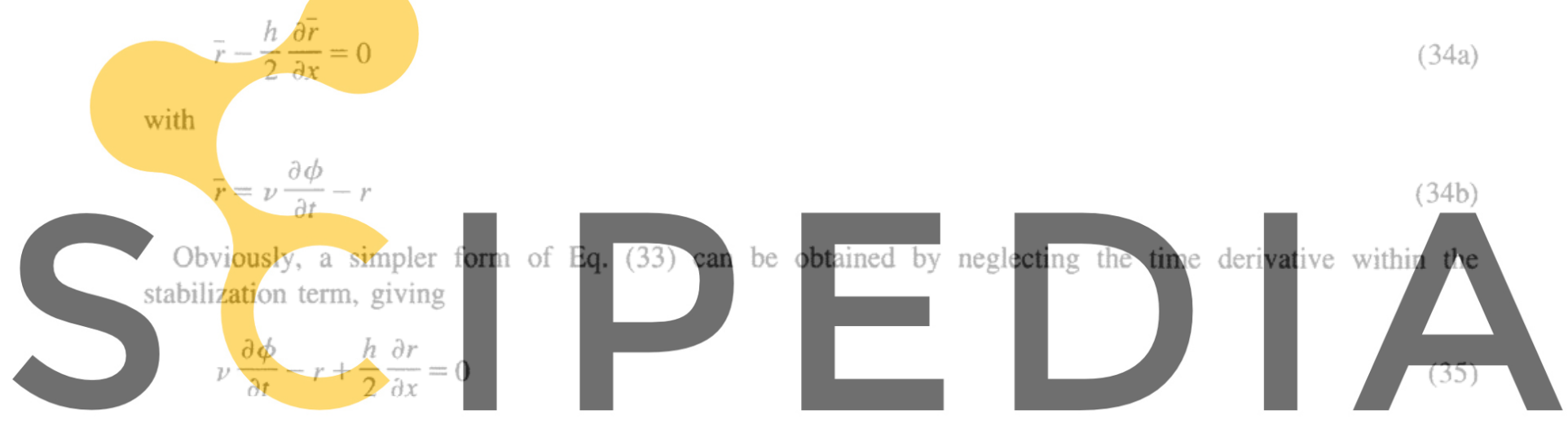

2.6. Equivalence with characteristic and Lax-Wendroff approximations

\section{Register for free at https//www.scipedia.com to download the version without the watermark \\ The explicit forward time integration of Eq. (35) reads}

$$
\Delta \phi=\frac{\Delta t}{\nu}\left[r-\tau u \frac{\partial r}{\partial x}\right]^{n}
$$

where $\Delta \phi=\phi^{n+1}-\phi^{n}$ and $h=2 \tau u$ has been taken.

Let us compare Eq. (36a) with the well established 'characteristic' and Lax-Wendroff approximations. The discretized equations in time for these two procedures can be expressed in identical form for the 1D case as $[3,21,22,23]$

$$
\Delta \phi=\frac{\Delta t}{\nu}\left[r-\frac{\Delta t}{2} u \frac{\partial r}{\partial x}\right]^{n}
$$

The equivalence between above two expressions is evident. In fact, both equations are identical if the intrinsic time $\tau$ in Eq. (36a) is taken equal to half the time increment within the brackets in Eq. (36b). The different meaning of the two time increments appearing in characteristic approximation schemes is now clear.

A simpler form of Eq. (36b) neglecting the diffusion flux in the balancing derivative term $\mathrm{d} r / \mathrm{d} x$ is typically used in the FEM with linear elements [3,11]. The full expression of Eq. (24) is however necessary if higher-order approximations are chosen as pointed out in [11].

\subsection{Equivalence with the Petrov-Galerkin FE formulation}

The standard Galerkin form for the FE solution of Eqs. (23) and (26) is equivalent to the so-called stabilized Petrov-Galerkin formulation. This equivalence is shown next. 
Let us construct first a finite element discretization $\left\{l^{e}\right\}$ of $l$ with index $e$ ranging from 1 to the number of elements $N_{e}$. The standard interpolation within an element $e$ with $n$ nodes can be written as [3]

$$
\phi \simeq \hat{\phi}=\sum_{i=1}^{n} N_{i} \phi_{i}
$$

where $N_{i}$ are the element shape functions and $\phi_{i}$ are nodal values of the approximate function $\hat{\phi}$. Substitution of Eq. (37) into Eqs. (23), (9) and (26) gives

$$
\begin{array}{ll}
\hat{r}-\frac{h}{2} \frac{\mathrm{d} \hat{r}}{\mathrm{~d} x}=r_{\Omega}, \quad 0 \leqslant x \leqslant l & \\
\hat{\phi}-\bar{\phi}=r_{\phi} & \text { in } x=0 \\
-\nu u \hat{\phi}+k \frac{\mathrm{d} \hat{\phi}}{\mathrm{d} x}+\bar{q}-\frac{h}{2} \hat{r}=r_{q} & \text { in } x=l
\end{array}
$$

where $\hat{r}=r(\hat{\phi})$ and $r_{\Omega}, r_{\phi}$ and $r_{q}$ are the residuals of the approximate solution in the domain and the Dirichlet and Neumann boundaries, respectively.

The weighted residual expression of Eqs. (38) is

$$
\int_{0}^{1} W r_{\Omega} \mathrm{d} \Omega+\left[\bar{W} r_{q}\right]_{x=1}+\left[\overline{\bar{W}} r_{\phi}\right]_{x=0}=0
$$

or

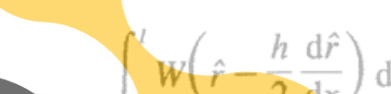

where $W, W$ and $\overline{\bar{W}}$ are appropriate

boundary at $x=0$, which implies satisfaction of the e

A simple integration by

$\int_{0}^{l}\left[W+\frac{h}{2} \frac{\mathrm{d} W}{\mathrm{~d} x}\right] \hat{r}$
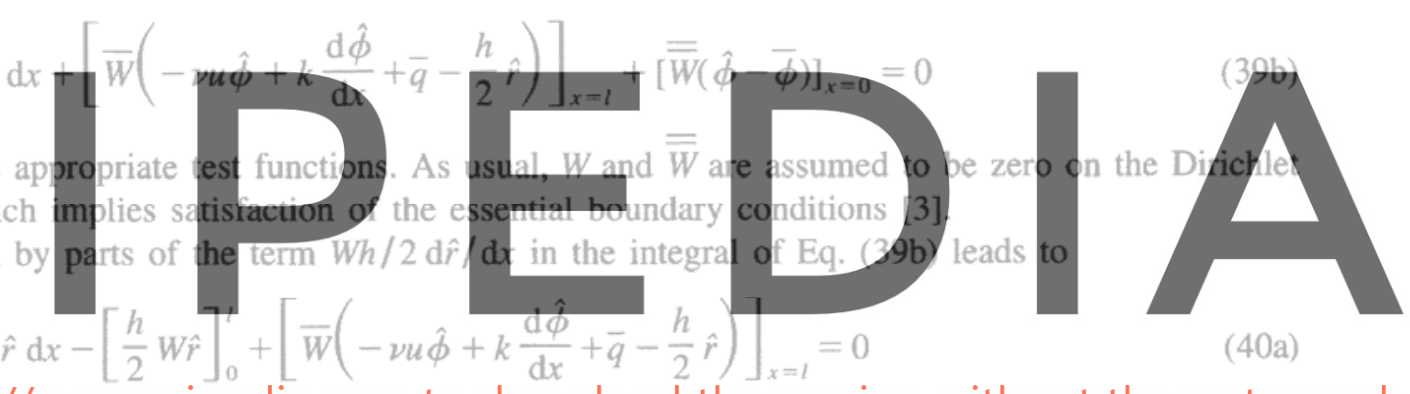

(40a)

Register for free at https//www.scipedia.com to download the version without the watermark

$$
\int_{0}^{l}\left[W+\frac{h}{2} \frac{\mathrm{d} W}{\mathrm{~d} x}\right] \hat{r} \mathrm{~d} x-\left[W\left(-\nu u \hat{\phi}+k \frac{\mathrm{d} \phi}{\mathrm{d} x}+\bar{q}\right)\right]_{x=l}=0
$$

Expression (40b) provides the final system of equations by making $W=W_{i}$ with index $i$ ranging from 1 to the number of nodes in the FE mesh. Note that by choosing $W_{i}=N_{i}$ the standard Petrov-Galerkin form used in FE analysis is recovered [3].

Integrating by parts the advective and diffusive terms in the product $W \hat{r}$ of Eq. (40b) and recalling again that $W=0$ in $x=0$ gives finally

$$
\begin{gathered}
\int_{0}^{l}\left[-\frac{\mathrm{d} W}{\mathrm{~d} x}(\nu u \hat{\phi})+\frac{\mathrm{d} W}{\mathrm{~d} x} k \frac{\mathrm{d} \hat{\phi}}{\mathrm{d} x}\right] \mathrm{d} x+\int_{0}^{l} \frac{h}{2} \frac{\mathrm{d} W}{\mathrm{~d} x}\left[\nu \frac{\mathrm{d}(u \hat{\phi})}{\mathrm{d} x}-\frac{\mathrm{d}}{\mathrm{d} x}\left(k \frac{\mathrm{d} \hat{\phi}}{\mathrm{d} x}\right)\right] \mathrm{d} x \\
=\int_{0}^{l} W Q \mathrm{~d} x-\bar{q}[W]_{l}+\int_{0}^{l} \frac{h}{2} \frac{\mathrm{d} W}{\mathrm{~d} x} Q \mathrm{~d} x \quad i=1, N_{e}
\end{gathered}
$$

With $W_{i}=N_{i}$ and $h=2 \tau u$ the one-dimensional version of the so-called Streamline Upwind Petrov-Galerkin (SUPG) formulation is readily recognized [1]. Also note that for $h=0$ the standard Galerkin form is recovered [3].

REMARK 8 . The choice of the length $h / 2$ for the balance domain next to the Neumann boundary appears now necessary to ensure the vanishing of the term $W \hat{r}$ on the boundary, thus recovering the standard Petrov-Galerkin formulation [3]. Recall that the value $h / 2$ coincides also with the distance traveled by a particle for a value of the intrinsic time $\tau=1$. 
REMARK 9. Integration by parts of the advective term in the expression of $W \hat{r}$ is not necessary if only the diffusive flux is prescribed at the Neumann boundary. The first integral in the l.h.s. of Eq. (40) is now given by

$$
\int_{0}^{l}\left[W \nu \frac{\mathrm{d}(u \phi)}{\mathrm{d} x}+\frac{\mathrm{d} W}{\mathrm{~d} x} k \frac{\mathrm{d} \phi}{\mathrm{d} x}\right] \mathrm{d} x
$$

whereas all remaining terms in Eq. (40) remain unchanged. Note that the final system of equations is the same in both cases.

REMARK 10. A consistent Petrov-Galerkin expression can be simply obtained for the transient case by starting with the stabilized transient form (34a). The resulting integral expression is identical to Eq. (40b) with the residual including now the time derivative term as in Eq. (34b).

REMARK 11. The second integral in the l.h.s. of Eq. (40c) can be expressed as

$$
\int_{0}^{l} \frac{h}{2} \frac{\mathrm{d} W}{\mathrm{~d} x}\left[\nu \frac{\mathrm{d}(\boldsymbol{u} \hat{\boldsymbol{\phi}})}{\mathrm{d} x}-\frac{\mathrm{d}}{\mathrm{d} x}\left(k \frac{\mathrm{d} \hat{\boldsymbol{\phi}}}{\mathrm{d} x}\right)\right] \mathrm{d} x=\sum_{e} \int_{l(e)} \frac{h}{2} \frac{\mathrm{d} W}{\mathrm{~d} x}\left[\nu \frac{\mathrm{d}(\boldsymbol{u} \hat{\boldsymbol{\phi}})}{\mathrm{d} x}-\frac{\mathrm{d}}{\mathrm{d} x}\left(k \frac{\mathrm{d} \hat{\boldsymbol{\phi}}}{\mathrm{d} x}\right)\right] \mathrm{d} x
$$

where the sum extends to all the elements in the mesh. Clearly the r.h.s. of Eq. (41b) allows to use $C_{0}$ continuous FE interpolations while preserving the consistency of the method. Obviously, the same elemental splitting can be applied to the stabilization integral affecting the source $Q$ in the r.h.s. of Eq. (40c) although this is not strictly necessary to allow for $C_{0}$ continuity. This elemental form of computing the stabilization integrals is widely used in practice $[5-7,10-12,15-19,24,26,29]$.

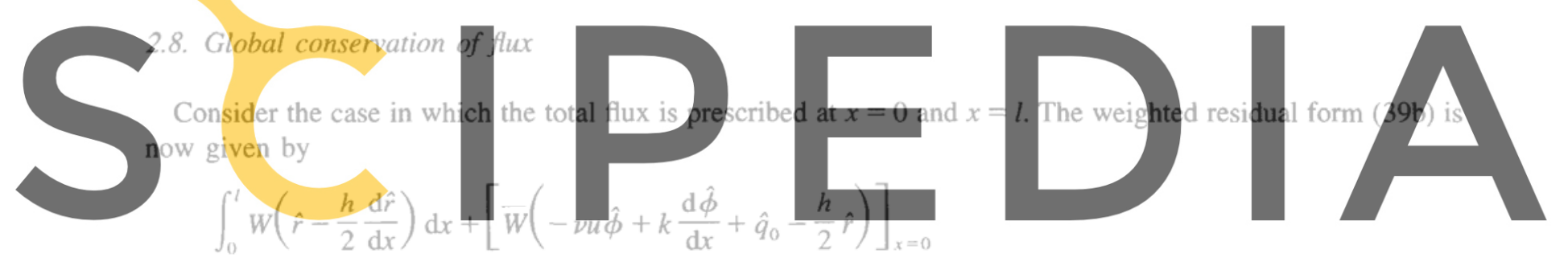

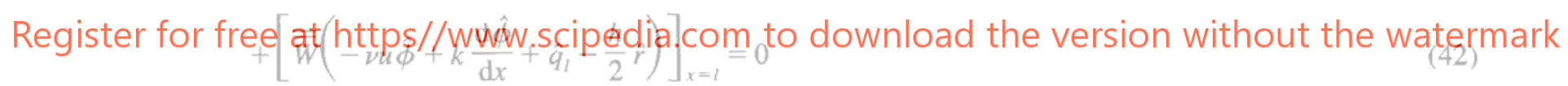

where $\hat{q}_{0}$ and $\hat{q}_{l}$ are the prescribed values of the total incoming and outgoing fluxes at $x=0$ and $x=l$, respectively. Following the same integration by parts of Section 2.6 gives after setting $W=\bar{W}=-\overline{\bar{W}}$

$$
\begin{gathered}
\int_{0}^{l}\left[-\frac{\mathrm{d} W}{\mathrm{~d} x}(\nu u \hat{\phi})+\frac{\mathrm{d} W}{\mathrm{~d} x} k \frac{\mathrm{d} \hat{\phi}}{\mathrm{d} x}\right] \mathrm{d} k+\int_{0}^{l} W \frac{h}{2} \frac{\mathrm{d} W}{\mathrm{~d} x}\left[\frac{\mathrm{d}(\nu u \hat{\phi})}{\mathrm{d} x}-\frac{\mathrm{d}}{\mathrm{d} x}\left(k \frac{\mathrm{d} \hat{\phi}}{\mathrm{d} x}\right)\right] \mathrm{d} x \\
=\int_{0}^{l} W Q \mathrm{~d} x-\bar{q}_{0}[W]_{0}-\bar{q}_{l}[W]_{l}+\int_{0}^{l} \frac{h}{2} \frac{\mathrm{d} W}{\mathrm{~d} x} Q \mathrm{~d} x
\end{gathered}
$$

Let us chose now $W=1$. This gives

$$
\int_{0}^{l} Q \mathrm{~d} x+\bar{q}_{0}-\bar{q}_{l}=0
$$

which confirms the conservation property of the case assumed.

\section{Two-dimensional advection-diffusion problems}

\subsection{Basic stabilized equation}

The concepts of previous section will be extended now to the solution of the advection-diffusion problem in a two-dimensional domain $\Omega$ with boundary $\Gamma$. Let us consider a finite rectangular domain of dimensions $h_{x}$ and 


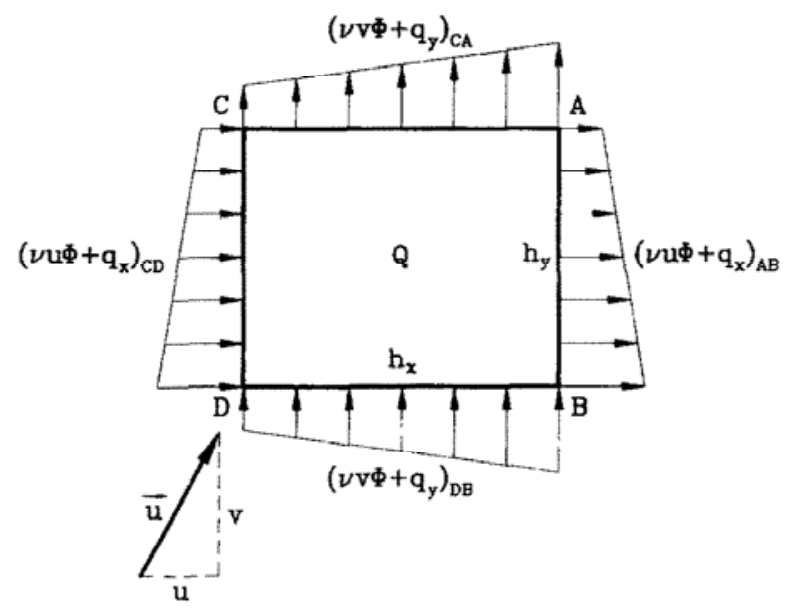

Fig. 4. Balance domain for 2D advection-diffusion problem. Advective and diffusive fluxes are assumed to vary linearly along the sides.

$h_{y}$ in directions $x$ and $y$, respectively. Both the advective and diffusive fluxes are assumed to vary linearly along the four sides (Fig. 4). The flux balance equation will be obtained using the following Taylor expansions:

\section{diffusive term: third-order expansion}

advective term: third-order expansion

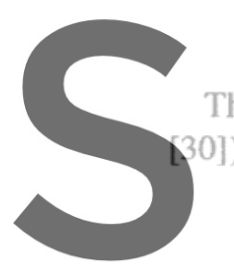

\section{source term:}

The balance of flux across the fo 0])
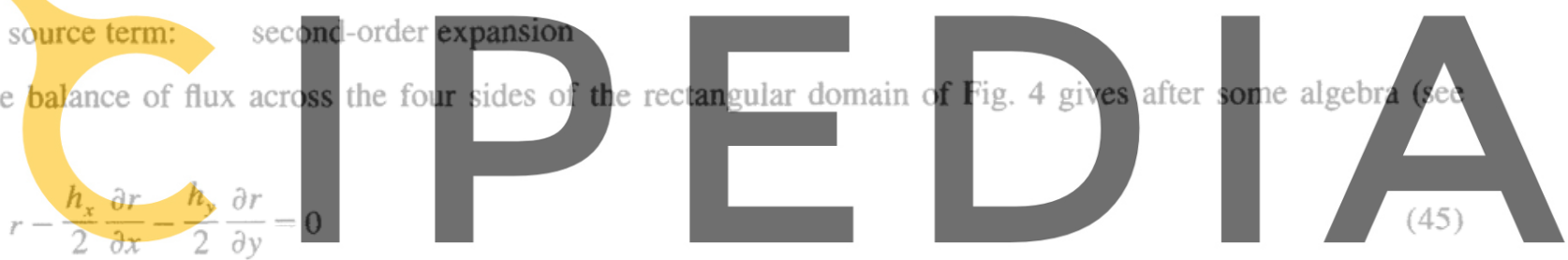

Register for $r=-\nu \nabla^{\mathrm{T}} f+\nabla^{\mathrm{T}}(D \nabla \phi)+Q$. where

$$
\boldsymbol{f}=[u \phi, v \phi]^{\mathrm{T}}
$$

is the advective flux vector,

$$
\boldsymbol{\nabla}=\left[\frac{\partial}{\partial x}, \frac{\partial}{\partial y}\right]^{\mathrm{T}}
$$

is the gradient operator, and

$$
\boldsymbol{D}=\left[\begin{array}{cc}
k_{x} & 0 \\
0 & k_{y}
\end{array}\right]
$$

is the conductivity matrix. For simplicity $x$ and $y$ are assumed to coincide here with the principal axes of material orthotropy. In Eq. (46) $\nu$ is the advective flux parameter which will be assumed to be constant throughout the domain $\Omega$ ( $\nu=\rho c$ for thermal convection problems). Also, as usual the velocities $u$ and $v$ are taken as positive if coincident with the direction of the global $x$ and $y$ axes, respectively.

Eq. (45) can be rewritten as

$$
r-\frac{1}{2} h^{\mathrm{T}} \nabla r=0 \quad \text { in } \Omega
$$

with

$$
\boldsymbol{h}=\left[h_{x}, h_{y}\right]^{\mathrm{T}}
$$


The boundary conditions are written as

$$
\phi-\bar{\phi}=0 \text { on } \Gamma_{\phi}
$$

where $\Gamma_{\phi}$ is the Dirichlet boundary, where the variable is prescribed, and

$$
-\nu \boldsymbol{n}^{\mathrm{T}} \boldsymbol{u} \phi+\boldsymbol{n}^{\mathrm{T}} \boldsymbol{D} \boldsymbol{\nabla} \phi+\bar{q}_{n}-\frac{1}{2} \boldsymbol{h}^{\mathrm{T}} \boldsymbol{n} \boldsymbol{r}=0 \quad \text { on } \Gamma_{4}
$$

where $\bar{q}_{n}$ is the prescribed total flux across the Neumann boundary $\Gamma_{q}$ with $\Gamma=\Gamma_{\phi} \cup \Gamma_{q}$ and $n=\left[n_{x}, n_{y}\right]^{\mathrm{T}}$ is the normal vector.

Eq. (52) has been obtained by balance of fluxes in the boundary domain of Fig. 5 following the same procedure of the 1D case previously explained (see [30]).

Note that the advective term $-\nu \boldsymbol{n}^{\mathrm{T}} \boldsymbol{u} \phi$ is dropped in Eq. (52) in the case when only the diffusive flux across the Neumann boundary is prescribed.

The stabilized transient form of Eq. (41) is obtained using the same arguments of Section 2.5 for the 1D case (see [30])

$$
\nu \frac{\partial \phi}{\partial t}-r-\frac{1}{2} h^{\mathrm{T}} \nabla\left(\nu \frac{\partial \phi}{\partial t}-r\right)=0
$$

with the initial condition $\phi=\phi_{0}$ at time $t=t_{0}$.

The standard differential equations are simply obtained neglecting the stabilizing terms in Eqs. (50)-(51) (i.e. making $h=0$ ) which gives for the steady state case

$$
\begin{array}{ll}
r=0 & \text { in } \Omega \\
\phi-\bar{\phi}=0 & \text { on } \Gamma_{\phi} \\
-\boldsymbol{\nu} \boldsymbol{n}^{\mathrm{T}} \boldsymbol{u} \phi+\boldsymbol{n}^{\mathrm{T}} \boldsymbol{D} \boldsymbol{\nabla} \phi+\bar{q}_{n}=0 & \text { on } \Gamma_{q}
\end{array}
$$

The extension to three-dimensional problems is straightforward and identical stabilized expressions are obtained.

\subsection{The concept of intrinsic time in $2 D$ advective-diffusive problems}

It can be further assumed that $h_{x}=h \cos \alpha$ and $h_{y}=h \sin \alpha$, where $\alpha$ is the angle which the velocity vector $\boldsymbol{u}=[u, v]^{\mathrm{T}}$ forms with the global $x$ axis and the distance $h=\left(h_{x}^{2}+h_{y}^{2}\right)^{1 / 2}$ is the characteristic length of the 2D advective-diffusive problem.

The stabilized governing equations (50) and (53) can now be written as

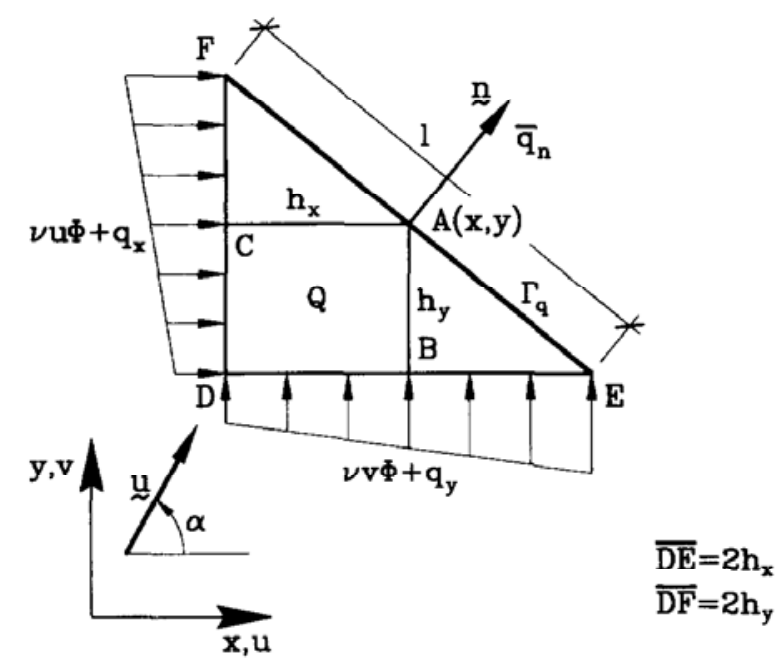

Fig. 5. Balance of fluxes in a two-dimensional domain next to a Neumann boundary. 
Steady state

$$
r-\frac{h}{2|\boldsymbol{u}|} \boldsymbol{u}^{\mathrm{T}} \nabla r=0
$$

Transient

$$
\nu \frac{\partial \phi}{\partial t}-r-\frac{h}{2|\boldsymbol{u}|} \boldsymbol{u}^{\mathrm{T}} \boldsymbol{\nabla}\left(\nu \frac{\partial \phi}{\partial t}-r\right)=0
$$

In the derivation of Eqs. (55) use of the identities $\sin \alpha=u /|\boldsymbol{u}|$ and $\cos \alpha=v /|\boldsymbol{u}|$ has been made.

The intrinsic time in 2D advective-diffusive problems is defined now as [5]

$$
\tau=\frac{h}{2|u|}
$$

With this definition Eqs. (55) can be rewritten as

Steady state

$$
r-\pi u^{\mathrm{T}} \nabla r=0 \quad \text { in } \Omega
$$

Transient

$$
\nu \frac{\partial \phi}{\partial t}-r-\tau \boldsymbol{u}^{\mathrm{T}} \nabla\left(\nu \frac{\partial \phi}{\partial t}-r\right)=0 \quad \text { in } \Omega
$$

The stabilized Neumann boundary condition (52) can also be expressed in terms of the intrinsic time as

$$
\begin{array}{ll}
-\boldsymbol{\nu} \boldsymbol{n}^{\mathrm{T}} \boldsymbol{u} \phi+\boldsymbol{n}^{\mathrm{T}} \boldsymbol{D} \boldsymbol{\nabla} \phi+\bar{q}_{n}-\tau \boldsymbol{u}^{\mathrm{T}} \boldsymbol{n} \boldsymbol{r}=0 & \text { on } \Gamma_{q} \text {, or } \\
-\boldsymbol{\nu} \boldsymbol{n}^{\mathrm{T}} \boldsymbol{u} \phi+\boldsymbol{n}^{\mathrm{T}} \boldsymbol{D} \boldsymbol{\nabla} \phi+\bar{q}_{n}-\frac{h}{2|\boldsymbol{u}|} \boldsymbol{u}^{\mathrm{T}} \boldsymbol{n} r=0 & \text { on } \Gamma_{q}
\end{array}
$$

\subsection{Equivalence with characteristic and Lax-Wendroff approximations}

Let us consider the stabilized transient equation (57b) neglecting for simplicity the effect of the time derivative in the stabilization term. A simple forward integration in time gives

$$
\Delta \phi=\frac{\Delta t}{\nu}\left[r-\tau u^{\mathrm{T}} \nabla r\right]^{n}
$$

The characteristic approximation for $2 \mathrm{D}$ problems is [22]

$$
\Delta \phi=\frac{\Delta t}{\nu}\left[r-\frac{\Delta t}{2} u^{\mathrm{T}} \nabla r\right]^{n}
$$

The equivalence between Eqs. (59) and (60) is obvious if the time increment $\Delta t$ between the brackets in Eq. (60) is taken to coincide with half the intrinsic time $\tau$ as in the 1D case.

The same equivalence can be found with the Lax-Wendroff approximation in two dimensions which can be expressed in a form identical to Eq. (61) $[1,21]$.

\subsection{Equivalence with the Petrov-Galerkin and SUPG formulations in the FEM}

Substituting the finite element approximation (37) into Eqs. (55a), (51) and (58b) gives

$$
\begin{array}{lr}
\hat{r}-\frac{h}{2|\boldsymbol{u}|} \boldsymbol{u}^{\mathrm{T}} \boldsymbol{\nabla} \hat{r}=r_{\Omega} & \text { in } \Omega \\
\hat{\boldsymbol{\phi}}-\overline{\boldsymbol{\phi}}=r_{\phi} & \text { on } \Gamma_{\phi} \\
-\boldsymbol{\nu} \boldsymbol{n}^{\mathrm{T}} \boldsymbol{u} \hat{\boldsymbol{\phi}}+\boldsymbol{n}^{\mathrm{T}} \boldsymbol{D} \boldsymbol{\nabla} \hat{\boldsymbol{\phi}}+\bar{q}_{n}-\frac{h}{2|\boldsymbol{u}|} \boldsymbol{u}^{\mathrm{T}} \boldsymbol{n} \hat{r}=r_{q} & \text { on } \Gamma_{q}
\end{array}
$$


where $\hat{r}=r(\hat{\phi})$ and $r_{\Omega}, r_{\phi}$ and $r_{q}$ are the solution residuals in the domain and the Dirichlet and Neumann boundaries, respectively.

The weighted residual form of Eqs. (61) is

$$
\begin{aligned}
& \iint_{\Omega} W\left(\hat{r}-\frac{h}{2|\boldsymbol{u}|} \boldsymbol{u}^{\mathrm{T}} \boldsymbol{\nabla} \hat{r}\right) \mathrm{d} \Omega+\int_{\Gamma_{q}} \bar{W}\left(-\boldsymbol{\nu} \boldsymbol{n}^{\mathrm{T}} \boldsymbol{u} \hat{\boldsymbol{\phi}}+\boldsymbol{n}^{\mathrm{T}} \boldsymbol{D} \boldsymbol{\nabla} \hat{\boldsymbol{\phi}}+\bar{q}_{n}-\frac{h}{2|\boldsymbol{u}|} \boldsymbol{u}^{\mathrm{T}} \boldsymbol{n} \hat{r}\right) \mathrm{d} \Gamma \\
& \quad+\int_{\Gamma_{\phi}} \overline{\bar{W}}(\hat{\boldsymbol{\phi}}-\bar{\phi}) \mathrm{d} \Gamma=0
\end{aligned}
$$

where $W, \bar{W}$ and $\overline{\bar{W}}$ are arbitrary test functions. As usual $W=\overline{\bar{W}}=0$ on $\Gamma_{\phi}$ will be assumed, thus implying exact satisfaction of the essential boundary conditions at the Dirichlet boundary.

Integrating by parts the term including $\nabla \hat{r}$ in the first integral of Eq. (62) gives

$$
\begin{aligned}
& \iint_{\Omega}\left(W+\frac{h}{2|\boldsymbol{u}|} \boldsymbol{u}^{\mathrm{T}} \nabla W\right) \hat{r} \mathrm{~d} \Omega-\int_{\Gamma} W \frac{h}{2|\boldsymbol{u}|} \boldsymbol{u}^{\mathrm{T}} \boldsymbol{n} \hat{r} \mathrm{~d} \Gamma \\
& \quad+\int_{\Gamma_{q}} \bar{W}\left(-\boldsymbol{\nu} \boldsymbol{n}^{\mathrm{T}} \boldsymbol{u} \hat{\phi}+\boldsymbol{n}^{\mathrm{T}} \boldsymbol{D} \boldsymbol{\nabla} \hat{\phi}+\bar{q}_{n}-\frac{h}{2|\boldsymbol{u}|} \boldsymbol{u}^{\mathrm{T}} \boldsymbol{n} \hat{r}\right) \mathrm{d} \Gamma=0
\end{aligned}
$$

Choosing now $\bar{W}=-W$ and recalling that $W=0$ on $\Gamma_{\phi}$ gives

$$
\iint_{\Omega}\left(W+\frac{h}{2|\boldsymbol{u}|} \boldsymbol{u}^{\mathrm{T}} \nabla W\right) \hat{r} \mathrm{~d} \Omega-\int_{\Gamma_{q}} W\left(-\boldsymbol{\nu} \boldsymbol{n}^{\mathrm{T}} \boldsymbol{u} \hat{\phi}+\boldsymbol{n}^{\mathrm{T}} \boldsymbol{D} \nabla \hat{\boldsymbol{\phi}}+\bar{q}_{n}\right) \mathrm{d} \Gamma=0
$$

The discrete system of equations is readily obtained making $W=W_{i}$ with $i$ ranging from 1 to the number of nodes in the FE mesh. Choosing now $W_{i}=N_{i}$ the Petrov-Galerkin form widely used in FE analysis is recognized [3].

Integrating by parts the advective and diffusive terms in the product $W \hat{r}$ of Eq. (64) and recalling again that $W=0$ on $\Gamma_{\phi}$ gives

$$
\begin{gathered}
\iint_{\Omega}\left[-\left(\nabla^{\mathrm{T}} W\right) \nu \hat{f}+\left(\boldsymbol{\nabla}^{\mathrm{T}} W\right) \boldsymbol{D} \boldsymbol{\nabla} \hat{\boldsymbol{\phi}}\right] \mathrm{d} \Omega+\iint_{\Omega} \frac{h}{2|\boldsymbol{u}|} \boldsymbol{u}^{\mathrm{T}} \nabla W\left[\nu \boldsymbol{\nabla}^{\mathrm{T}} \boldsymbol{f}-\boldsymbol{\nabla}^{\mathrm{T}}(\boldsymbol{D} \nabla \hat{\boldsymbol{\phi}})\right] \mathrm{d} \Omega \\
=\iint_{\Omega} W Q \mathrm{~d} \Omega+\iint_{\Omega} \frac{h}{2|\boldsymbol{u}|} \boldsymbol{u}^{\mathrm{T}}(\boldsymbol{\nabla} W) Q \mathrm{~d} \Omega-\int_{\Gamma_{4}} W \bar{q}_{n} \mathrm{~d} \Gamma
\end{gathered}
$$

where $\hat{f}=\boldsymbol{f}(\hat{\phi})$. With $W_{i}=N_{i}\left(i=1, N_{e}\right)$ and $h=2 \tau|\boldsymbol{u}|$ the well-known Streamline Upwind Petrov-Galerkin (SUPG) expression used in FE computations is obtained [3,5]. Obviously, the same arguments of Remark 11 can be used in this case to allow for $C_{0}$ continuous $\mathrm{FE}$ interpolations.

An identical expression to Eq. (64) is obtained for the transient case if the time derivative term is included in the residual similarly as described in Section 2.5 for the $1 \mathrm{D}$ case.

CONCLUSION. The Galerkin solution of the stabilized problem governed by Eqs. (62) is equivalent to the Petrov-Galerkin (PG) and SUPG formulations of the standard problem posed by Eqs. (54). The well-known stabilization properties of the PG and SUPG approaches would therefore carry forward to the Galerkin solution of the (equivalent) new stabilized problem. In addition, a meaningful interpretation of the stabilization terms has been obtained. Also note that for $h=0$ the standard Galerkin form is recovered.

REMARK 12. The integration by parts of the advective terms in the expression of $W \hat{r}$ is not necessary if only the diffusive flux is prescribed at the Neumann boundary. The first integral in the l.h.s. of Eq. (65) is expressed now by

$$
\int_{\Omega}\left[W \nu \nabla^{\mathrm{T}} \hat{\boldsymbol{f}}+\left(\boldsymbol{\nabla}^{\mathrm{T}} W\right) \boldsymbol{D} \boldsymbol{\nabla} \hat{\boldsymbol{\phi}}\right] \mathrm{d} \Omega
$$

whereas all remaining terms of Eq. (65) remain unchanged. Note that the final system of equations is the same in both cases. 
It is also easy to verify following the procedure of Section 2.8 that global conservation of fluxes is satisfied.

\section{Stabilization of fluid flow equations. Basic concepts}

The same concepts of previous sections will be applied now to derive the stabilized form of the governing equations of fluid mechanics. The basic idea is to apply the standard conservation equations of fluid flow derived from Newton's second law, i.e. the momentum equations

$$
b=\frac{\partial}{\partial t} \int_{V} \rho u \mathrm{~d} V+\int_{A}(\rho u) u^{\mathrm{T}} \mathrm{d} A
$$

to a finite control domain.

In Eq. (67) $b$ is the vector containing the total forces acting over the control domain with volume $V$ bounded by a control surface with area $A$. Obviously, in the steady state case the time derivative is zero in Eq. (67). The equation of conservation of mass can be viewed as a particular case of Eq. (67) with $\boldsymbol{b}=0$ and $\rho \boldsymbol{u}=\rho$, i.e. the convected field is the density of the fluid.

In order to clarify concepts, let us consider the stationary flow balance equation over an one-dimensional duct segment $A B$ of length $h$ with $x_{B}=x$ and $x_{A}=x-h$ and transverse cross section of area A (Fig. 6). The application of Eq. (67) to this simple case is

$$
N_{B}+\int_{V} b \mathrm{~d} V-N_{A}=A[u(\rho u)]_{B}-A[u(\rho u)]_{A}
$$

where $N_{A}$ and $N_{B}$ are the viscous axial forces acting at the ends of the balance domain and $b$ are distributed axial forces. Note that in (68) $N_{B} \equiv N(x) \equiv N, N_{A} \equiv N(x-h)$ and the same applies to the term $u(\rho u)$.

The following approximations can be made

$$
\begin{aligned}
& N(x-h)=N(x)-h \frac{\mathrm{d} N}{\mathrm{~d} x}+\frac{h^{2}}{2} \frac{\mathrm{d}^{2} N}{\mathrm{~d} x^{2}}-\mathrm{O}\left(h^{3}\right) \\
& {[u(\rho u)](x-h)=[u(\rho u)](x)-h \frac{\mathrm{d}}{\mathrm{d} x}[u(\rho u)]+\frac{h^{2}}{2} \frac{\mathrm{d}^{2}}{\mathrm{~d} x^{2}}[u(\rho u)]-\mathrm{O}\left(h^{3}\right)} \\
& \int_{V} b \mathrm{~d} V=\frac{A h}{2}[b(x)+b(x-h)]=A h b(x)-\frac{A h^{2}}{2} \frac{\mathrm{d} b}{\mathrm{~d} x}+\mathrm{O}\left(h^{2}\right)
\end{aligned}
$$

Substitution of Eqs. (69) into Eq. (68) gives after simplification

$$
r-\frac{h}{2} \frac{\mathrm{d} r}{\mathrm{~d} x}=0
$$

with

$$
r=-\frac{\mathrm{d}}{\mathrm{d} x}[u(\rho u)+p]+\frac{\mathrm{d}}{\mathrm{d} x}\left(\mu \frac{\mathrm{d} u}{\mathrm{~d} x}\right)+b
$$

In the derivation of Eqs. (70) and (71) use of the constitutive relationship $N=A(\mu \mathrm{d} u / \mathrm{d} x-p)$, where $\mu$ is

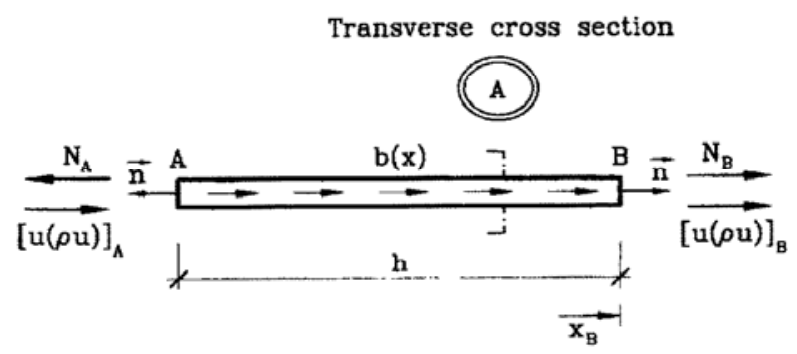

Fig. 6. Duct segment where balance of momentum is imposed. 
the fluid viscosity and $p$ is the pressure has been made. Eq. (70) coincides with the standard 1D momentum equation when $h=0$. The distance $h$ is the characteristic length of the stabilized fluid flow problem governed now by Eq. (70).

Let us chose now a segment of length $h / 2$ next to the duct exit and where a traction force is applied (see Fig. 7). Using similar arguments of Section 2.4 leads to the following stabilized form of the Neumann boundary condition as

$$
\mu \frac{\mathrm{d} u}{\mathrm{~d} x}-p-\bar{N}-\frac{h}{2} r=0 \quad \text { on } x=l
$$

where $\bar{N}$ is the applied traction force at the duct end.

The transient form of Eq. (70) can be readily written using Eq. (67) as

$$
\frac{\partial(\rho u)}{\partial t}-r-\frac{h}{2} \frac{\partial}{\partial x}\left(\frac{\partial(\rho u)}{\partial t}-r\right)=0
$$

In the derivation of Eq. (73) use of the same arguments of Section 2.5 to evaluate the time derivative integral has been made.

Similar arguments can be used to derive the following stabilized forms of the equations of mass and energy conservation.

Mass conservation:

$$
\frac{\partial \rho}{\partial t}+\frac{\partial(\rho u)}{\partial x}-\frac{h}{2} \frac{\partial}{\partial x}\left(\frac{\partial \rho}{\partial t}+\frac{\partial(\rho u)}{\partial x}\right)=0
$$

Energy conservation:

$$
\frac{\partial(\rho E)}{\partial t}-r_{E}-\frac{h}{2} \frac{\partial}{\partial x}\left(\frac{\partial(\rho E)}{\partial t}-r_{E}\right)=0
$$

with

$$
r_{E}=-\frac{\partial}{\partial x}(\rho E+p) u+\frac{\partial}{\partial x}\left(k \frac{\partial T}{\partial x}+u \mu \frac{\mathrm{d} u}{\mathrm{~d} x}\right)+Q
$$

In Eq. (75) the quantity $E$ represents the total energy given by the sum of the thermal and kinetic energies

$$
E=c_{v} T+\frac{1}{2} u^{2}
$$

where $T$ is the temperature and $c_{v}$ represents the heat capacity at constant volume. For a thermally and calorically perfect gas the relationship between pressure, density and temperature is given by the universal gas law

$$
p=p R T
$$

where $R$ is the gas constant. The quantities $Q$ and $k$ in Eq. (76) represent the distributed heat source per unit volume and the thermal conductivity, respectively.

Above equations have been derived for the general case of an one-dimensional compressible fluid. The particular stabilized forms of the momentum and mass conservation equation for an isothermal incompressible fluid $(\rho=$ constant $)$ can be easily obtained as

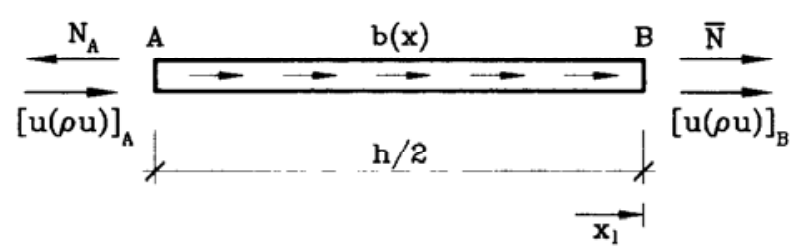

Fig. 7. Balance of fluxes in a duct segment next to a Neumann boundary. 


\section{Momentum}

$$
\rho \frac{\partial u}{\partial t}-r-\frac{h}{2} \frac{\partial}{\partial x}\left(\rho \frac{\partial u}{\partial t}-r\right)=0
$$

with

$$
r=-\rho u \frac{\partial u}{\partial x}-\frac{\partial p}{\partial x}+\frac{\partial}{\partial x}\left(\mu \frac{\partial u}{\partial x}\right)+b
$$

Incompressibility

$$
\frac{\partial u}{\partial x}-\frac{h}{2} \frac{\partial^{2} u}{\partial x^{2}}=0
$$

These equations are completed by the stabilized Neumann boundary condition (72) and the standard Dirichlet condition of prescribed velocity at the entry point.

\section{Extension to two- and three-dimensional fluid flow problems}

The extension of above stabilization procedure to two- and three-dimensional fluid flow problems is straightforward. The basic principle in all cases is the satisfaction of the balance equation in a finite domain assuming a third-order Taylor expansion for the convection and viscous terms and a linear expansion for the body forces. The resulting stabilized expressions of the governing equations for compressible and incompressible fluids will be shown next.

\subsection{Stabilization of compressible flow equations}

The proposed stabilization procedure leads to the following conservation form of the three-dimensional mass conservation, momentum and energy equations [3]

$$
\frac{\partial \boldsymbol{v}}{\partial t}-\boldsymbol{r}-\frac{1}{2} \boldsymbol{H}^{k} \frac{\partial}{\partial x_{k}}\left(\frac{\partial \boldsymbol{v}}{\partial t}-\boldsymbol{r}\right)=0 \quad k=1,2,3
$$

where

$$
\boldsymbol{v}=\left[\rho, \rho u_{1}, \rho u_{2}, \rho u_{3}, \rho E\right]^{\mathrm{T}}
$$

is the vector of unknown variables, where as usual $\rho$ is the density, $u_{i}$ is the velocity in the $i$ th direction and $E$ is the total energy. Also in Eq. (82)

$$
\boldsymbol{r}=-\frac{\partial f_{i}}{\partial x_{i}}-\frac{\partial g_{i}}{\partial x_{i}}-q
$$

where

$$
f_{i}=\left[\rho u_{i}, \rho u_{1} u_{i}+\delta_{1 i} p, \rho u_{2} u_{i}+\delta_{2 i} p, \rho u_{3} u_{i}+\delta_{3 i} p, u_{i}(\rho E+p)\right]^{\mathrm{T}}
$$

defines the convective flux vector and

$$
g_{i}=\left[0,-\tau_{1 i},-\tau_{2 i},-\tau_{3 i},-k_{i} \frac{\partial T}{\partial x_{i}}-\tau_{i j} u_{j}\right]^{\mathrm{T}}
$$

defines the diffusion fluxes. Finally

$$
\boldsymbol{q}=\left[0,-b_{1},-b_{2},-b_{3},-b_{i} u_{i}-Q\right]^{\mathrm{T}}
$$

gives the source terms due to body forces $\rho f_{i}$ (i.e. gravity forces) and a distributed heat source $Q$. In above $p$ is 
the pressure, $T$ is the temperature, $E$ is the total energy and $\tau_{i j}$ are the viscous stress components related to the velocity gradients by

$$
\tau_{i j}=\mu\left(\frac{\partial u_{i}}{\partial x_{j}}+\frac{\partial u_{j}}{\partial x_{i}}-\frac{2}{3} \frac{\partial u_{k}}{\partial x_{k}} \delta_{i j}\right)
$$

The relationship between pressure, density and temperature is given by the universal gas law Eq. (78). Matrix $\boldsymbol{H}_{k}$ appearing in the stabilizing term of Eq. (82) can be obtained as

$$
\boldsymbol{H}^{k}=\left[\begin{array}{cccccc}
h_{\rho}^{k} & & & & & \mathbf{0} \\
& h_{u}^{k} & & & \\
& & h_{u}^{k} & & \\
& & & h_{u}^{k} & \\
& & & & h_{e}^{k}
\end{array}\right] ; \quad k=1,2,3
$$

In above $h_{\rho}^{i}, h_{u}^{i}, h_{e}^{i}, i=1,2,3$ are the dimensions of the right prism where mass, momentum and energy balances are enforced, respectively. Note that for $\boldsymbol{H}_{k}=0$ the standard conservation form of the compressible flow equation is recovered as

$$
\frac{\partial v}{\partial t}-r=0
$$

i.e.

$$
\frac{\partial \boldsymbol{v}}{\partial t}+\frac{\partial \boldsymbol{f}_{i}}{\partial x_{i}}+\frac{\partial \boldsymbol{g}_{i}}{\partial x_{i}}+\boldsymbol{q}=0
$$

\subsection{Definition of characteristic lengths, intrinsic times and stabilized traction boundary conditions}

The following characteristic lengths for each of the five equations governing mass, momentum and energy balances can be now defined as

$$
\begin{aligned}
& h_{\rho}=\left|\boldsymbol{h}_{\rho}\right| \quad \text { with } \boldsymbol{h}_{\rho}=\left[h_{\rho}^{1}, h_{\rho}^{2}, h_{\rho}^{3}\right]^{\mathrm{T}} \\
& h_{u}=\left|\boldsymbol{h}_{u}\right| \quad \text { with } \boldsymbol{h}_{u}=\left[h_{u}^{1}, h_{u}^{2}, h_{u}^{3}\right]^{\mathrm{T}} \\
& h_{e}=\left|\boldsymbol{h}_{e}\right| \quad \text { with } \boldsymbol{h}_{e}=\left[h_{e}^{1}, h_{e}^{2}, h_{e}^{3}\right]^{\mathrm{T}}
\end{aligned}
$$

It will now be assumed that the characteristic length vectors $\boldsymbol{h}_{\rho}, \boldsymbol{h}_{u}$ and $\boldsymbol{h}_{e}$ have all the direction of the velocity vector $\boldsymbol{u}=\left[u_{1}, u_{2}, u_{3}\right]^{\mathrm{T}}$. This allows us to express matrix $\boldsymbol{H}_{k}$ as

$$
\boldsymbol{H}^{k}=\frac{u_{k}}{|\boldsymbol{u}|} \boldsymbol{H} \quad \text { with } \boldsymbol{H}=\left[\begin{array}{llllll}
h_{\rho} & & & & & 0 \\
& h_{u} & & & \\
& & h_{u} & & \\
& & & h_{u} & \\
\mathbf{0} & & & & h_{e}
\end{array}\right]
$$

and the stabilized equations have now the following form

$$
\frac{\partial \boldsymbol{v}}{\partial t}-\boldsymbol{r}-\frac{u_{k}}{2|\boldsymbol{u}|} \boldsymbol{H} \frac{\partial}{\partial x_{k}}\left(\frac{\partial \boldsymbol{v}}{\partial t}-\boldsymbol{r}\right)=0
$$

The intrinsic time for each of the balancing equations can be defined as

$$
\tau_{\rho}=\frac{h_{\rho}}{2|\boldsymbol{u}|}, \quad \tau_{u}=\frac{h_{u}}{2|\boldsymbol{u}|}, \quad \tau_{e}=\frac{h_{e}}{2|\boldsymbol{u}|}
$$

Eq. (93) can be rewritten using (94) as 


$$
\frac{\partial \boldsymbol{v}}{\partial t}-\boldsymbol{r}-[\boldsymbol{\tau}] u_{k} \frac{\partial}{\partial x_{k}}\left(\frac{\partial \boldsymbol{v}}{\partial t}-\boldsymbol{r}\right)=0
$$

where the intrinsic time matrix is

$$
[\boldsymbol{\tau}]=\left[\begin{array}{ccccc}
\tau_{\rho} & & & & \mathbf{0} \\
& \tau_{u} & & & \\
& & \tau_{u} & & \\
& & & \tau_{u} & \\
\mathbf{0} & & & & \tau_{e}
\end{array}\right]
$$

Similar higher-order balance concepts can be applied to a finite domain next to a Neumann boundary $\Gamma_{t}$ where tractions $\bar{t}$ are specified (see Fig. 8). The resulting stabilized form of the traction boundary condition can be written as (see [30])

$$
\boldsymbol{M \sigma}-\bar{t}-\frac{1}{2} \boldsymbol{n}^{\mathrm{T}} \boldsymbol{h}_{u} \boldsymbol{r}_{m}=\mathbf{0}
$$

where $\boldsymbol{n}$ is the normal vector

$$
\boldsymbol{\sigma}=\left[\sigma_{11}, \sigma_{22}, \sigma_{33}, \sigma_{12}, \sigma_{13}, \sigma_{23}\right]^{\mathrm{T}}
$$

with

$$
\begin{aligned}
\sigma_{i j} & =\tau_{i j}-p \delta_{i j} \\
\boldsymbol{M} & =\left[\begin{array}{ccccccc}
n_{x} & 0 & 0 & n_{y} & n_{z} & 0 \\
0 & n_{y} & 0 & n_{x} & 0 & n_{z} \\
0 & 0 & n_{z} & 0 & n_{x} & n_{y}
\end{array}\right]
\end{aligned}
$$

and $r_{m}$ contains the momentum equations, i.e. the rows 2, 3 and 4 of vector $\boldsymbol{r}$ of Eq. (84).

Eq. (97) can be rewritten in terms of the characteristic length $h_{u}$ using Eqs. (91b) and (92) as

$$
\boldsymbol{M} \boldsymbol{\sigma}-\bar{t}-\frac{h_{u}}{2|\boldsymbol{u}|} \boldsymbol{n}^{\top} \boldsymbol{u} \boldsymbol{r}_{m}=\mathbf{0}
$$

Indeed, for boundaries where the outgoing heat flux is specified the stabilized form given by Eq. (52) should be used.

The equivalent stabilized equations (82), (93) and (95) are the basis for the numerical solution of

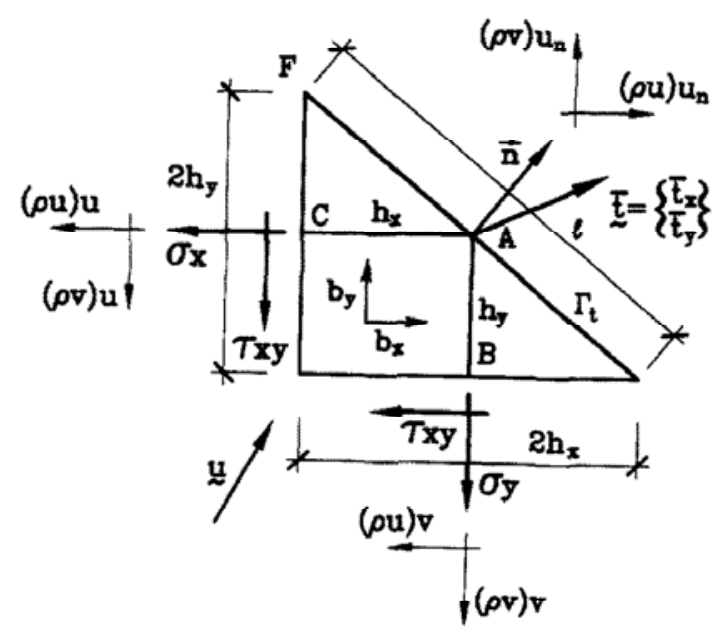

Fig. 8. Flow domain next to a Neumann boundary where surface tractions are prescribed. 
compressible flow problems using FD, FV, FE or FP procedures. As usual, the time integration can be performed prior to the application of any spatial discretization scheme. Neglecting the time derivative in the stabilization term and using a simple forward integration gives the following equivalent expressions

$$
\Delta \boldsymbol{v}=\Delta t\left[\boldsymbol{r}-\frac{1}{2} \boldsymbol{H}^{k} \frac{\partial \boldsymbol{r}}{\partial x_{k}}\right]^{n}=\Delta t\left[\boldsymbol{r}-\frac{u_{k}}{2|\boldsymbol{u}|} \boldsymbol{H} \frac{\partial \boldsymbol{r}}{\partial x_{k}}\right]^{n}=\Delta t\left[\boldsymbol{r}-[\boldsymbol{\tau}] u_{k} \frac{\partial \boldsymbol{r}}{\partial x_{k}}\right]^{n}
$$

where $\Delta v=\boldsymbol{v}^{n+1}-\boldsymbol{v}^{n}$.

The analogy of above expressions with the characteristic approximation and the Petrov-Galerkin scheme is shown in next section.

REMARK 13. The assumption that all the characteristic length vectors are oriented in the direction of the velocity vector $\boldsymbol{u}$ is not strictly necessary. Other possibilities can therefore be explored, starting with the basic form of the stabilized equations (82).

REMARK 14. The derivation of the optimum characteristic length (or the intrinsic time) parameter for each of the governing flow equations is usually based on simplified analogies with the one-dimensional advectivediffusive equation [3]. An alternative procedure is suggested in Section 8.

\subsection{Equivalence with the characteristic approximation}

Note that Eqs. (82), (93) and (95) involve a different characteristic length or intrinsic timescale for each of the governing differential equations. A simpler form can be derived assuming $\boldsymbol{h}_{\rho}=\boldsymbol{h}_{u}=\boldsymbol{h}_{e}=\boldsymbol{h}$, i.e. the balancing domain is taken to have equal dimensions for each of the governing equations. The equivalent stabilized equations (82), (93) and (95) can be written as

$$
\begin{aligned}
& \frac{\partial \boldsymbol{v}}{\partial t}-\boldsymbol{r}-\frac{1}{2} \overline{\boldsymbol{H}}^{k} \frac{\partial}{\partial x_{k}}\left(\frac{\partial \boldsymbol{v}}{\partial t}-\boldsymbol{r}\right)=0 \\
& \frac{\partial \boldsymbol{v}}{\partial t}-\boldsymbol{r}-\frac{h}{2|\boldsymbol{u}|} u_{k} \frac{\partial}{\partial x_{k}}\left(\frac{\partial \boldsymbol{v}}{\partial t}-\boldsymbol{r}\right)=0 \\
& \frac{\partial \boldsymbol{v}}{\partial t}-\boldsymbol{r}-\tau u_{k} \frac{\partial}{\partial x_{k}}\left(\frac{\partial \boldsymbol{v}}{\partial t}-\boldsymbol{r}\right)=0
\end{aligned}
$$

where

$$
\begin{aligned}
& h=|\boldsymbol{h}| \quad \text { with } \boldsymbol{h}=\left[h^{1}, h^{2}, h^{3}\right]^{\mathbf{T}} \\
& \overline{\boldsymbol{H}}^{k}=h^{k} \boldsymbol{I}_{5} \quad \text { and } \quad \tau=\frac{h}{2|\boldsymbol{u}|}
\end{aligned}
$$

where $\boldsymbol{I}_{5}$ is the $5 \times 5$ unit matrix.

The explicit integration of Eq. (102c) gives (neglecting the time derivative in the stabilization term)

$$
\Delta \boldsymbol{v}=\Delta t\left[\boldsymbol{r}-\tau u_{k} \frac{\partial \boldsymbol{r}}{\partial x_{k}}\right]^{n}
$$

Following the arguments given in [11] the characteristic approximation of the compressible Navier-Stokes equations can be found as $[3,22,23]$

$$
\Delta \boldsymbol{v}=\Delta t\left[\boldsymbol{r}-\frac{\Delta t}{2} u_{k} \frac{\partial \boldsymbol{r}}{\partial x_{k}}\right]^{n}
$$

The equivalence between above two expressions is clear if the time increment between the brackets in Eq. (105) is taken to be equal to twice the intrinsic time $\tau$ of Eq. (104). Therefore, any of the two approximations can be used as starting point for the solution in space using a standard Galerkin FE scheme. Indeed, the more general expressions (102) can be used to derive a (possibly) more advantageous alternative FE scheme. 


\subsection{Equivalence with the Lax-Wendroff approximation}

The explicit time discretization of the standard compressible flow equations can be written as

$$
\Delta v=\Delta t\left[r-\frac{\Delta t}{2} \frac{\partial}{\partial x_{k}} A_{k} r\right]^{n}
$$

where

$$
\boldsymbol{A}_{k}=\frac{\partial f_{k}}{\partial \boldsymbol{v}}
$$

Note that the form of Eqs. (104) and (106a) is very similar. The full equivalence occurs only if the time increment between the brackets in Eq. (106a) is twice the intrinsic time of Eq. (104) and the gradient matrix $\boldsymbol{A}_{k}=u_{k} \boldsymbol{I}_{5}$, where $\boldsymbol{I}_{5}$ is the $5 \times 5$ unit matrix and $u_{k}$ is a constant velocity. This coincidence is unusual and, in general, the numerical solution of Eqs. (104) and (106a) will be different [1,21].

\subsection{Equivalence with Petrov-Galerkin and SUPG finite element forms}

Let us assume now a standard FE approximation written as

$$
\boldsymbol{v} \simeq \hat{\boldsymbol{v}}=\sum_{i=1}^{n_{e}} N_{i} \boldsymbol{v}_{i}
$$

where $\boldsymbol{v}_{i}$ are nodal values of the approximate solution $\hat{\boldsymbol{v}}$. Substituting the FE approximation (107) into Eq. (102b) gives (for the stationary case)

$$
\hat{\boldsymbol{r}}-\frac{h}{2|\boldsymbol{u}|} u_{k} \frac{\partial \hat{\boldsymbol{r}}}{\partial x_{k}}=r_{\Omega} \quad \text { in } \Omega
$$

where $\hat{\boldsymbol{r}}=\boldsymbol{r}(\hat{\boldsymbol{v}})$.

For simplicity isothermal conditions will be assumed. Also, only Neumann boundaries $\Gamma_{t}$ where surface tractions are specified via Eq. (100) will be considered (here for simplicity $h_{u}=h$ will be taken). All other boundaries will be assumed to be of Dirichlet type where the essential boundary conditions in velocities are exactly satisfied at element nodes. The weighted residual form of Eqs. (100) and (108) can therefore be written as

$$
\int_{\Omega} \boldsymbol{W}\left[\hat{\boldsymbol{r}}-\frac{h}{2|\boldsymbol{u}|} u_{k} \frac{\partial \hat{\boldsymbol{r}}}{\partial x_{k}}\right] \mathrm{d} \Omega+\int_{\Gamma_{t}} \overline{\boldsymbol{W}}\left[\boldsymbol{M} \boldsymbol{\sigma}-\overline{\boldsymbol{t}}-\frac{h}{2|\boldsymbol{u}|} \boldsymbol{n}^{\mathrm{T}} \boldsymbol{u} \hat{\boldsymbol{r}}_{m}\right] \mathrm{d} \Gamma=0
$$

where $\hat{r}$ excludes now the energy equation. In Eq. (109) $\boldsymbol{W}$ and $\bar{W}$ are diagonal matrices containing arbitrary weighting functions. Integrating by parts the term involving $\partial \hat{r} / \partial x_{k}$ in the first integral of Eq. (109) and making $\bar{W}=-W_{3}$ where $\boldsymbol{W}_{3}$ contains the rows and columns 2,3 and 4 of matrix $\boldsymbol{W}$, gives after some simplifications

$$
\int_{\Omega}\left(\boldsymbol{W}+\frac{h}{2|\boldsymbol{u}|} u_{k} \frac{\partial \boldsymbol{W}}{\partial x_{k}}\right) \hat{\boldsymbol{r}} \mathrm{d} \Omega-\int_{\Gamma_{t}} \boldsymbol{W}_{3}(\boldsymbol{M} \boldsymbol{\sigma}-\overline{\boldsymbol{t}}) \mathrm{d} \Gamma=0
$$

The discrete systems of equations is readily obtained making $\boldsymbol{W}=\boldsymbol{W}_{i}$. Choosing now $\boldsymbol{W}_{i}=N_{i} \boldsymbol{I}_{5}$ in Eq. (110) where $\boldsymbol{I}_{5}$ is the $4 \times 4$ unit matrix, the standard Petrov-Galerkin form used in FE analysis is found [3]. Again the arguments of Remark 11 can be now used to allow for $C_{0} \mathrm{FE}$ interpolations.

An identical expression to Eq. (110) is obtained for the transient case if the time derivative terms of Eq. $(102 b)$ are included in the residual.

\subsection{Equivalence with Laplacian pressure operator schemes}

It is interesting to note the equivalence of the stabilization procedure described above and numerical schemes based on the stabilization of the continuity equation by adding a Laplacian pressure operator $[3,11,22,23]$. This equivalence is shown next for the steady state case. 
Let us rewrite the stabilized form of the continuity equation using Eq. (102c) as

$$
\frac{\partial\left(\rho u_{i}\right)}{\partial x_{i}}-\tau_{\rho} u_{k} \frac{\partial}{\partial x_{k}}\left(\frac{\partial\left(\rho u_{i}\right)}{\partial x_{i}}\right)=0
$$

where

$$
\tau_{\rho}=\frac{h_{\rho}}{2|\boldsymbol{u}|} .
$$

It is easy to prove that

$$
u_{k} \frac{\partial}{\partial x_{k}}\left(\frac{\partial\left(\rho u_{i}\right)}{\partial x_{i}}\right)=\frac{\partial}{\partial x_{k}}\left(u_{k} \frac{\partial\left(\rho u_{i}\right)}{\partial x_{i}}\right)-\frac{\mathrm{d} u_{k}}{\mathrm{~d} x_{k}} \frac{\mathrm{d}\left(\rho u_{i}\right)}{\mathrm{d} x_{i}}
$$

The original momentum equation can be written as

$$
\frac{\partial}{\partial x_{i}}\left(\rho u_{j} u_{i}+\delta_{j i} p\right)-\frac{\partial \tau_{j i}}{\partial x_{i}}-\rho f_{i}=0 \quad i, j=1,3
$$

or

$$
\left(\rho u_{i}\right) \frac{\partial u_{j}}{\partial x_{i}}+u_{j} \frac{\partial\left(\rho u_{i}\right)}{\partial x_{i}}+\delta_{j i} \frac{\partial p}{\partial x_{i}}-\frac{\partial \tau_{j i}}{\partial x_{i}}-\rho f_{j}=0
$$

Differentiating this equation gives

$$
\frac{\partial}{\partial x_{j}}\left(u_{j} \frac{\partial\left(\rho u_{i}\right)}{\partial x_{i}}\right)=-\delta_{j i} \frac{\partial^{2} p}{\partial x_{i} x_{j}}-\frac{\partial}{\partial x_{j}}\left(\left(\rho u_{i}\right) \frac{\partial u_{j}}{\partial x_{i}}\right)+\frac{\partial^{2} \tau_{j i}}{\partial x_{i} \partial x_{j}}+\frac{\partial}{\partial x_{j}}\left(\rho f_{j}\right)=0
$$

Substituting Eqs. (112) and (114) into (111) and neglecting terms such as those underlined in Eqs. (112) and (114) gives

$$
\frac{\partial\left(\rho u_{i}\right)}{\partial x_{i}}+\tau_{\rho} \Delta p-\tau_{\rho} \frac{\partial}{\partial x_{j}}\left(\rho f_{j}\right)=0
$$

where

$$
\Delta=\left(\frac{\partial}{\partial x^{2}}+\frac{\partial}{\partial y^{2}}\right)
$$

is the Laplacian operator and the last term, of Eq. (115) is due to body forces. Assuming these to be uniform gives

$$
\frac{\partial\left(\rho u_{i}\right)}{\partial x_{i}}+\tau_{\rho} \Delta p=0
$$

i.e. the stabilized form of the continuity equation (111) can be interpreted as the addition of a Laplacian pressure operator to the original mass conservation equation.

Eq. (116) naturally also holds for the case of constant density (incompressible flow) giving simply

$$
\frac{\partial u_{i}}{\partial x_{i}}+\frac{\tau_{\rho}}{\rho} \Delta p=0
$$

Clearly Eqs. (116)-(118) are advantageous to derive FE schemes using equal order interpolation thus circumventing the LBB condition in the limit incompressible case [3,11]. The equivalence between Eqs. (111) and (116) opens various possibilities for deriving alternative div-stable FE schemes where the advantages of the new stabilized form can be exploited. 


\section{Stabilization of incompressible flow equations}

Assuming the flow to be isothermal and the density to be constant in Eqs. (102) gives the following stabilized form of the incompressible flow equations.

Continuity equation

$$
\frac{\partial u_{i}}{\partial x_{i}}-\frac{h_{\rho}^{k}}{2} \frac{\partial}{\partial x_{k}}\left(\frac{\partial u_{i}}{\partial x_{i}}\right)=0
$$

Momentum equations

$$
\frac{\partial \boldsymbol{u}}{\partial t}-\boldsymbol{r}_{m}-\frac{1}{2} \boldsymbol{H}_{m}^{k} \frac{\partial}{\partial x_{k}}\left(\frac{\partial \boldsymbol{u}}{\partial t}-\boldsymbol{r}_{m}\right)=0
$$

where

$$
\begin{aligned}
& \boldsymbol{u}=\left[u_{1}, u_{2}, u_{3}\right]^{\mathrm{T}}, \quad \boldsymbol{H}_{m}^{k}=h_{u}^{k} \boldsymbol{I}_{3} \\
& \boldsymbol{r}_{m}=-\left[\nabla \boldsymbol{u}^{\mathrm{T}}\right] \boldsymbol{u}-\frac{1}{\rho} \nabla p+\frac{1}{\rho} \boldsymbol{L}^{\mathrm{T}} \boldsymbol{\sigma}+\boldsymbol{b}
\end{aligned}
$$

with

$$
\begin{aligned}
& \boldsymbol{b}=\left[f_{1}, f_{2}, f_{3}\right]^{\mathrm{T}} ; \quad \boldsymbol{\nabla}=\left[\frac{\partial}{\partial x}, \frac{\partial}{\partial y}, \frac{\partial}{\partial z}\right]^{\mathrm{T}} \\
& \boldsymbol{L}^{\mathrm{T}}=\left[\begin{array}{cccccc}
\partial / \partial x & 0 & 0 & \partial / \partial y & \partial / \partial z & 0 \\
0 & \partial / \partial y & 0 & \partial / \partial x & 0 & \partial / \partial z \\
0 & 0 & \partial / \partial z & 0 & \partial / \partial x & \partial / \partial y
\end{array}\right]
\end{aligned}
$$

and the expression of $\boldsymbol{\sigma}$ is given by Eq. (98a).

Eqs. (118) and (119) can be rewritten in terms of the characteristic lengths and the intrinsic times by using the following expressions

$$
\begin{aligned}
& h_{\rho}^{k}=\frac{h_{\rho}}{2|\boldsymbol{u}|} u_{k}=\tau_{\rho} u_{k} \\
& h_{u}^{k}=\frac{h_{u}}{2|\boldsymbol{u}|} u_{k}=\tau_{u} u_{k}
\end{aligned}
$$

Note also that the stabilized continuity equation (118) can be replaced by the more standard equivalent form (117) obtained using the same procedure of Section 5.6.

\section{Higher-order stabilization scheme}

\subsection{One-dimensional advective-diffusive problem}

A higher-order stabilization scheme can be simply derived by using higher-order expansions in the approximation of the flux and source terms in the balancing domain. For simplicity, let us consider first the one-dimensional advection-diffusion problem. The balance equation over the typical segment $A B$ of Fig. 1(b) can be written following the notation of Section 2.1 as

$$
q(x)+\nu[u \phi](x)-q(x-h)-\nu[u \phi](x-h)+\frac{h}{6}\left[Q(x)+4 Q\left(x-\frac{h}{2}\right)+Q(x-h)\right]=0
$$

In Eq. (123) a quadratic distribution of the external source $Q$ has been taken.

The following expansions can be written 


$$
\begin{aligned}
& q(x-h)=q(x)-h \frac{\mathrm{d} q}{\mathrm{~d} x}+\frac{h^{2}}{2} \frac{\mathrm{d}^{2} q}{\mathrm{~d} x^{2}}-\frac{h^{3}}{6} \frac{\mathrm{d}^{3} q}{\mathrm{~d} x^{3}}+\frac{h^{4}}{24} \frac{\mathrm{d}^{4} q}{\mathrm{~d} x^{4}}+\mathrm{O}\left(h^{5}\right) \\
& {[u \phi](x-h)=[u \phi](x)-h \frac{\mathrm{d}[u \phi]}{\mathrm{d} x}+\frac{h^{2}}{2} \frac{\mathrm{d}^{2}[u \phi]}{\mathrm{d} x^{2}}-\frac{h^{3}}{6} \frac{\mathrm{d}^{3}[u \phi]}{\mathrm{d} x^{3}}+\frac{h^{4}}{24} \frac{\mathrm{d}^{4}[u \phi]}{\mathrm{d} x^{4}}+\mathrm{O}\left(h^{5}\right)} \\
& Q\left(x-\frac{h}{2}\right)=Q(x)-\frac{h}{2} \frac{\mathrm{d} Q}{\mathrm{~d} x}+\frac{h^{2}}{8} \frac{\mathrm{d}^{2} Q}{\mathrm{~d} x^{2}}-\frac{h^{2}}{48} \frac{\mathrm{d}^{3} Q}{\mathrm{~d} x^{3}}-\mathrm{O}\left(h^{4}\right) \\
& Q(x-h)=Q(x)-h \frac{\mathrm{d} Q}{\mathrm{~d} x}+\frac{h^{2}}{2} \frac{\mathrm{d}^{2} Q}{\mathrm{~d} x^{2}}-\frac{h^{3}}{6} \frac{\mathrm{d}^{3} Q}{\mathrm{~d} x^{3}}-\mathrm{O}\left(h^{4}\right)
\end{aligned}
$$

Substituting Eqs. (124) into (123) gives after some simple algebra

$$
r-\frac{h}{2} \frac{\mathrm{d} r}{\mathrm{~d} x}+\frac{h^{2}}{6} \frac{\mathrm{d}^{2} r}{\mathrm{~d} x^{2}}-\frac{h^{3}}{24} \frac{\mathrm{d}^{3} r}{\mathrm{~d} x^{3}}=0
$$

where $r$ is given by Eq. (24). Note that Eq. (125) incorporates naturally the higher-order stabilization terms

$$
\frac{h^{2}}{6} \frac{\mathrm{d}^{2} r}{\mathrm{~d} x^{2}} \text { and } \frac{h^{3}}{24} \frac{\mathrm{d}^{3} r}{\mathrm{~d} x^{3}} .
$$

The higher-order stabilized transient equation can be expressed in identical form to Eq. (125) simply by including the time derivative term in the residual as shown in Eqs. (34).

Eq. (125) can be expressed in term of the intrinsic time scale simply making $h=2 \tau u$. This gives

$$
r-\tau u \frac{\mathrm{d} r}{\mathrm{~d} x}+\frac{2}{3} \tau^{2} u^{2} \frac{\mathrm{d}^{2} r}{\mathrm{~d} x^{2}}-\frac{1}{3} \tau^{3} u^{3} \frac{\mathrm{d}^{3} r}{\mathrm{~d} x^{3}}=0
$$

A simple stabilized transient form neglecting the time derivative in the stabilization terms is

$$
\nu \frac{\partial \phi}{\partial t}-r+\tau u \frac{\mathrm{d} r}{\mathrm{~d} x}-\frac{2}{3} \tau^{2} u^{2} \frac{\mathrm{d}^{2} r}{\mathrm{~d} x^{2}}+\frac{1}{3} \tau^{3} u^{3} \frac{\mathrm{d}^{3} r}{\mathrm{~d} x^{3}}=0
$$

The explicit time integration of Eq. (127) is written as

$$
\Delta \phi=\frac{\Delta t}{\nu}\left[r-\tau u \frac{\mathrm{d} r}{\mathrm{~d} x}+\frac{2}{3} \tau^{2} u^{2} \frac{\mathrm{d}^{2} r}{\mathrm{~d} x^{2}}-\frac{1}{3} \tau^{3} u^{3} \frac{\mathrm{d}^{3} r}{\mathrm{~d} x^{3}}\right]^{n}
$$

Similar ideas can be used to derive higher-order stabilized forms of the Neumann boundary condition.

\subsection{Analogy with stabilization schemes based on adding second-and fourth-order diffusive terms}

The stabilization term involving the second derivatives of the residual in Eqs. (127) is usually not taken into account in FD, FE and FV schemes [1,3]. The reason for neglecting this term is that it appears multiplied by the square of the velocity. Here, this term is unable to capture the effect of a sign change in the velocity field. The resulting stabilized equation involving first- and third-order derivatives of the residual only is

$$
r-\frac{h}{2} \frac{\mathrm{d} r}{\mathrm{~d} x}-\frac{h^{3}}{24} \frac{\mathrm{d}^{3} r}{\mathrm{~d} x^{3}}=0
$$

Eqs. (129) can be interpreted as a generalization of the standard stabilization procedures based on adding second- and fourth-order diffusive terms to the original equation [1]. Indeed, taking into account the convective term only in the residual derivatives in Eqs. (129) gives

$$
r-\frac{h}{2} \frac{\mathrm{d}^{2}(u \phi)}{\mathrm{d} x^{2}}-\frac{h^{3}}{24} \frac{\mathrm{d}^{4}(u \phi)}{\mathrm{d} x^{4}}=0
$$


The analogy with a number of similar stabilized FD and FV schemes widely used for advective-diffusive transport and high speed flow situations is now clearly recognized [1].

\subsection{Higher-order stabilization of the two-dimensional advection-diffusion equations}

The extension to two-dimensional advective-diffusive problems is straightforward. For this purpose a quadratic variation of the fluxes along the sides of the rectangular balancing domain of Fig. 3 is assumed. Following the procedure described in [30] gives

$$
r-\nabla^{1} r+\nabla^{2} r-\nabla^{3} r=0
$$

where as usual $r$ is given by Eq. (24) and

$$
\begin{aligned}
& \boldsymbol{\nabla}^{1}=\frac{1}{2} \boldsymbol{h}^{\mathrm{T}} \boldsymbol{\nabla} \quad \text { with } \boldsymbol{h}=\left[h_{x}, h_{y}\right]^{\mathrm{T}}, \quad \boldsymbol{\nabla}=\left[\frac{\partial}{\partial x}, \frac{\partial}{\partial y}\right]^{T} \\
& \boldsymbol{\nabla}^{2}=\frac{h_{x}^{2}}{6} \frac{\partial^{2}}{\partial x^{2}}+\frac{h_{x} h_{y}}{4} \frac{\partial^{2}}{\partial x \partial y}+\frac{h_{y}^{2}}{6} \frac{\partial^{2}}{\partial y^{2}} \\
& \boldsymbol{\nabla}^{3}=\frac{h_{x}^{3}}{24} \frac{\partial^{3}}{\partial x^{3}}+\frac{h_{x}^{2} h_{y}}{12} \frac{\partial^{3}}{\partial x^{2} \partial y}+\frac{h_{x} h_{y}^{2}}{12} \frac{\partial^{3}}{\partial x \partial y^{2}}+\frac{h_{y}^{3}}{24} \frac{\partial^{3}}{\partial y^{3}}
\end{aligned}
$$

The transient form is identical to Eq. (131) if the residual is defined as in Eqs. (34) (see [30]). A simpler transient expression neglecting the time derivative in the stabilization terms is

$$
\nu \frac{\partial \phi}{\partial t}-r+\nabla^{1} r-\nabla^{2} r+\nabla^{3} r=0
$$

The explicit time integration of Eq. (133) gives

$$
\Delta \phi=\frac{\Delta t}{\nu}\left[r-\nabla^{1} r+\nabla^{2} r-\nabla^{3} r\right]
$$

A similar process can be followed to derive higher-order stabilization schemes for the fluid mechanics equations.

\subsection{Analogy with the Subgrid Scale and Galerkin-Least-Square forms}

Let us consider the following stabilized form of the one-dimensional convection-diffusion equation obtained by neglecting the $h^{3}$ stabilization term in Eq. (125), i.e.

$$
r-\frac{h}{2} \frac{\mathrm{d} r}{\mathrm{~d} x}+\frac{h^{2}}{6} \frac{\mathrm{d}^{2} r}{\mathrm{~d} x^{2}}=0
$$

This equation is completed with the Dirichlet boundary condition (9) and the stabilized Neumann condition given by Eq. (26).

The finite element solution of these equations will now be attempted. For that purpose the following weighted residual form will be written

$$
\int_{0}^{l} W\left[\hat{r}-\frac{h}{2} \frac{\mathrm{d} \hat{r}}{\mathrm{~d} x}+\frac{h^{2}}{6} \frac{\mathrm{d}^{2} \hat{r}}{\mathrm{~d} x^{2}}\right] \mathrm{d} x+\left[\bar{W}\left(-\nu u \hat{\phi}+k \frac{\mathrm{d} \hat{\phi}}{\mathrm{d} x}+\bar{q}-\frac{h}{2} \hat{r}\right)\right]_{l}+[\overline{\bar{W}}(\hat{\phi}-\bar{\phi})]_{0}=0
$$

where $\hat{r}=r(\hat{\phi}), \hat{\phi}$ being the approximate function (viz. Eq. (35)).

As usual $W=\bar{W}=0$ in the Dirichlet boundary is now taken. Integrating once by parts the term

$$
W \frac{h}{2} \frac{\mathrm{d} \hat{r}}{\mathrm{~d} x}
$$

and twice the term 


$$
W \frac{h^{2}}{6} \frac{\mathrm{d}^{2} \hat{r}}{\mathrm{~d} x^{2}}
$$

gives after choosing $\bar{W}=-W$

$$
\int_{0}^{l}\left[W+\frac{h}{2} \frac{\mathrm{d} W}{\mathrm{~d} x}+\frac{h^{2}}{6} \frac{\mathrm{d}^{2} W}{\mathrm{~d} x^{2}}\right] \hat{r} \mathrm{~d} x-\left[W\left(-\nu u \hat{\phi}+k \frac{\mathrm{d} \hat{\phi}}{\mathrm{d} x}+\bar{q}\right)\right]_{1}+\frac{h^{2}}{6}\left[\left(W \frac{\mathrm{d} \hat{r}}{\mathrm{~d} x}-\hat{r} \frac{\mathrm{d} W}{\mathrm{~d} x}\right)_{1}+\left(\hat{r} \frac{\mathrm{d} W}{\mathrm{~d} x}\right)_{a}\right]=0
$$

Expression (137) provides the system of equations in the FE solution by making $W=W_{i}$ with index $i$ ranging from 1 to the number of nodes in the FE mesh. Note that by choosing $W_{i}=N_{i}$ an expression analogous to that used by Franca et al. [10] and later by Hughes [15] with the name of Subgrid Scale (SS) method is recovered. This can be made more evident by integrating the advective and diffusive terms in the product $W \hat{r}$ giving

$$
\begin{gathered}
\int_{0}^{l}\left[\frac{\mathrm{d} W}{\mathrm{~d} x}(\nu u \hat{\phi})+\frac{\mathrm{d} W}{\mathrm{~d} x} k \frac{\mathrm{d} \hat{\phi}}{\mathrm{d} x}\right] \mathrm{d} x+\int_{0}^{l}\left[\frac{h}{2} \frac{\mathrm{d} W}{\mathrm{~d} x}+\frac{h^{2}}{6} \frac{\mathrm{d}^{2} W}{\mathrm{~d} x^{2}}\right]\left[\frac{\mathrm{d}(\nu u \hat{\phi})}{\mathrm{d} x}-\frac{\mathrm{d}}{\mathrm{d} x}\left(\hat{k} \frac{\mathrm{d} \hat{\phi}}{\mathrm{d} x}\right)\right] \mathrm{d} x \\
=\int_{0}^{l} W Q \mathrm{~d} x-\bar{q}[W]_{l}+\int_{0}^{l}\left(\frac{h}{2} \frac{\mathrm{d} W}{\mathrm{~d} x}+\frac{h^{2}}{6} \frac{\mathrm{d}^{2} W}{\mathrm{~d} x^{2}}\right) Q \mathrm{~d} x+\frac{h^{2}}{6}\left[\left(W \frac{\mathrm{d} \hat{r}}{\mathrm{~d} x}-\hat{r} \frac{\mathrm{d} W}{\mathrm{~d} x}\right)_{l}+\left(\hat{r} \frac{\mathrm{d} W}{\mathrm{~d} x}\right)_{0}\right]
\end{gathered}
$$

With $W_{i}=N_{i}$ and $h=2 \tau u$, Eq. (138) reproduces the features of the SS formulation [15,29]. This approach is very similar to the so-called Galerkin-Least-Square method [24], the only difference being the sign of the viscous operator applied to the test functions in the stabilization term. A similar stabilized term is obtained with the Taylor-Galerkin (TG) procedure as shown in [29] where an interesting comparison of SUPG, SS, GLS and TG methods is presented.

A similar analogy can be found for two-dimensional problems.

\section{Higher-order approximations in time}

Let us write the balance equation for the one-dimensional advection-diffusion problem for the space-time slab $[A, B] \times[t, t+\delta]$, where $[A, B]$ is the finite spacial domain of Fig. 1 and $\delta$ is an arbitrary (finite) time increment as

$$
\int_{t}^{t+\delta}\left(\sum \text { internal fluxes }\right) \mathrm{d} t=\int_{A}^{B}\left[\int_{t}^{t+\delta} \nu \mathrm{d} \phi\right] \mathrm{d} x
$$

The 1.h.s. of Eq. (139) can be approximated as (using the arguments of Section 2.3)

$$
\begin{aligned}
\int_{t}^{t+\delta}\left(\sum \text { internal fluxes }\right) \mathrm{d} t & =\frac{\delta h}{2}\left[\left(r-\frac{h}{2} \frac{\partial r}{\partial x}\right)^{t}+\left(r-\frac{h}{2} \frac{\partial r}{\partial x}\right)^{t+\delta}\right] \\
& =\delta h\left[\left(r-\frac{h}{2} \frac{\partial r}{\partial x}\right)^{t}+\frac{\delta}{2} \frac{\partial}{\partial t}\left(r-\frac{h}{2} \frac{\partial r}{\partial x}\right)^{t}\right]
\end{aligned}
$$

where as usual $r$ is given by Eq. (24).

On the other hand the r.h.s. of Eq. (139) can be expressed as (taking $\nu$ as constant)

$$
\begin{aligned}
\int_{A}^{B}\left[\int_{t}^{t+\delta} \nu \mathrm{d} \phi\right] \mathrm{d} x & =\int_{A}^{B} \nu\left(\phi^{t+\delta}-\phi^{t}\right) \mathrm{d} x=\int_{A}^{B} \nu\left(\delta \frac{\partial \phi}{\partial t}+\frac{\delta^{2}}{2} \frac{\partial^{2} \phi}{\partial t^{2}}\right)^{t} \mathrm{~d} x \\
& =\nu \delta h\left[\left(\frac{\partial \phi}{\partial t}+\frac{\delta}{2} \frac{\partial^{2} \phi}{\partial t^{2}}\right)^{t}-\frac{h}{2} \frac{\partial}{\partial x}\left(\frac{\partial \phi}{\partial t}+\frac{\delta}{2} \frac{\partial^{2} \phi}{\partial t^{2}}\right)^{t}\right]
\end{aligned}
$$

Equaling now Eqs. (140) and (141) gives after simplification the final form of the stabilized transient balance equation as 


$$
\bar{r}-\frac{h}{2} \frac{\partial \bar{r}}{\partial x}+\frac{\delta}{2} \frac{\partial}{\partial t}\left(\bar{r}-\frac{h}{2} \frac{\partial \bar{r}}{\partial x}\right)=0
$$

where

$$
\bar{r}=\nu \frac{\partial \phi}{\partial t}-r
$$

In the derivation of Eq. (142a) $(\cdot)^{t}=(\cdot)$ has been taken. Note that by taking $\delta=h=0$ the standard infinitesimal form of the transient balance equation $\bar{r}=0$ is obtained. A simpler form of Eq. (142a) neglecting the high order term $\delta h$ is

$$
\bar{r}-\frac{h}{2} \frac{\partial \bar{r}}{\partial x}+\frac{\delta}{2} \frac{\partial \bar{r}}{\partial t}=0
$$

Eqs. (142a) and (143) can be used to derive time integration schemes where the characteristic time increment parameter $\delta$ could play an interesting stabilization role in some cases.

\section{Computation of the characteristic length parameter}

A popular method for deriving analytical expressions of the stabilization parameter $\alpha$ for 1D problems solved by the FEM is by adding some kind of artificial diffusion of the form

$$
\frac{\alpha u l^{(e)}}{2} \frac{\mathrm{d}^{2} \phi}{\mathrm{d} x^{2}}
$$

to the original differential equations and then computing $\alpha$ by imposing exact or quasi-exact nodal solution [3]. This can be shown to be equivalent to minimizing the truncation error of the numerical solution at each nodal point. This procedure can only be successfully applied for linear two node elements. For quadratic 1D elements two stabilization parameters are required to obtain exact nodal results, although a single average parameter can be used for practical purposes [23,24]. Extension of these concepts to 2D and 3D convective transport and flow problems is usually made on a simply heuristic grounds and no systematic method for deriving a general expression of the stabilization parameters is yet available. Recent attempts by Hughes [15] and Brezzi et al. [16-18] show some promise in this direction. In the next section an expression for computing the stabilization parameter is suggested.

\subsection{One-dimensional advection-diffusion problem}

The arguments somehow follow those of standard procedures mentioned above, i.e. the role of the stabilization parameter is to counterbalance the error introduced by the numerical algorithms.

For simplicity, let us consider the FE solution of the 1D advective-diffusive problem. The average residual of a particular numerical solution over an element can be defined as

$$
r^{(e)} \equiv \frac{1}{l^{(e)}} \int_{l^{(e)}} r_{\Omega} \mathrm{d} x
$$

where $r_{\Omega}$ is defined by Eq. (38a).

Substituting Eq. (38a) into (144) gives

$$
r^{(e)}=\hat{r}^{(e)}-\left(\frac{h}{2} \frac{\mathrm{d} \hat{r}}{\mathrm{~d} x}\right)^{(e)}
$$

where

$$
a^{(e)} \equiv \frac{1}{l^{(e)}} \int_{l^{(e)}} a \mathrm{~d} x
$$


For simplicity the value of $h$ will be assumed to be constant over the element, i.e. $h=h^{(e)}$. Hence, Eq. (145a) can be simplified to

$$
r^{(e)}=\hat{r}^{(e)}-\frac{h^{(e)}}{2}\left(\frac{\mathrm{d} \hat{r}}{\mathrm{~d} x}\right)^{(e)}
$$

Let us assume now that an enhanced numerical solution has been found for a given finite element mesh. This can be achieved by projecting into the original mesh an improved solution obtained via mesh refinement or, more simply, by global/local smoothing or superconvergent recovery of derivatives [27]. If $r_{1}^{(e)}$ and $r_{2}^{(e)}$, respectively, denote the element residuals of the original and the enhanced numerical solution for a given mesh then it is obvious that

$$
r_{1}^{(e)}-r_{2}^{(e)} \geqslant 0
$$

Obviously, both $r_{1}^{(e)}$ and $r_{2}^{(e)}$ have assumed to be positive in (147). In the case they are negative the above inequality should be changed to $\leqslant 0$.

Combining Eqs. (146) and (147) an expression for the element characteristic length can be found as

$$
h^{(e)} \geqslant 2\left(\hat{r}_{2}^{(e)}-\hat{r}_{1}^{(e)}\right)\left[\left(\frac{\mathrm{d} \hat{r}_{2}}{\mathrm{~d} x}\right)^{(e)}-\left(\frac{\mathrm{d} \hat{r}_{1}}{\mathrm{~d} x}\right)^{(e)}\right]^{-1}
$$

The equality case in (148) yields the critical value of the element characteristic length parameter ensuring no growth of the numerical error. The accuracy of expression (148) is shown next in a simple example.

\subsection{Example. The $1 D$ advective-diffusive problem}

Let us consider the FE solution of the 1D advection-diffusion problem

$$
-u \frac{\mathrm{d} \phi}{\mathrm{d} x}+k \frac{\mathrm{d}^{2} \phi}{\mathrm{d} x^{2}}=0, \quad 0 \leqslant x \leqslant l
$$

with boundary conditions

$$
\begin{array}{ll}
\phi=0 & \text { at } x=0 \\
\phi=1 & \text { at } x=1
\end{array}
$$

The solution will be attempted with the simplest two node linear element. For a uniform mesh the residual and the residual derivative for an element with nodes $i$ and $i+1$ can be found as

$$
\hat{r}_{1}^{(e)}=-\frac{u}{l^{(e)}}\left(\phi_{i+1}-\phi_{i}\right) \text { and }\left(\frac{\mathrm{d} \hat{r}_{1}}{\mathrm{~d} x}\right)^{(e)}=0
$$

The enhanced solution is obtained now by a simple smoothing of the first-order derivative at the nodes. The elemental residual for the enhanced solution is given by

$$
\hat{r}_{2}^{(e)}=-\frac{u}{2}\left(\hat{\phi}_{i}^{\prime}+\hat{\phi}_{i+1}^{\prime}\right)+\frac{k}{l^{(e)}}\left(\hat{\phi}_{i+1}^{\prime}-\hat{\phi}_{i}^{\prime}\right)
$$

where

$$
\hat{\phi}_{i}^{\prime}=\left(\frac{\mathrm{d} \hat{\phi}}{\mathrm{d} x}\right)_{i}=\frac{\phi_{i+1}-\phi_{i-1}}{2 l^{(e)}} .
$$

A simple algebra gives

$$
\hat{r}_{2}^{(e)}=-\frac{u}{4 l^{(e)}}\left(\phi_{i+1}-\phi_{i-1}+\phi_{i+2}-\phi_{i}\right)+\frac{k}{2\left(l^{(e)}\right)^{2}}\left(\phi_{i+2}-\phi_{i}-\phi_{i+1}+\phi_{i-1}\right)
$$


A similar procedure leads to

$$
\left(\frac{\mathrm{d} \hat{r}_{2}}{\mathrm{~d} x}\right)^{(e)}=-u \frac{\mathrm{d}^{2} \hat{\phi}}{\mathrm{d} x^{2}}=-\frac{u}{2\left(l^{(e)}\right)^{2}}\left[\phi_{i+2}-\phi_{i}-\phi_{i+1}+\phi_{i-1}\right]
$$

Substituting Eqs. (150), (152) and (153) into (148) gives the element critical characteristic length as

$$
h^{(e)}=\left[\frac{\phi_{i+2}-3 \phi_{i+1}+3 \phi_{i}-\phi_{i-1}}{\phi_{i+2}-\phi_{i+1}-\phi_{i}+\phi_{i-1}}-\frac{1}{\gamma}\right] l^{(e)}
$$

where $\gamma=u l^{(e)} / 2 k$ is the element Peclet number. The value of the element stabilization parameter $\alpha^{(e)}$ is readily deduced from Eq. (154) as

$$
\alpha^{(e)}=\frac{\phi_{i+2}-3 \phi_{i+1}+3 \phi_{i}-\phi_{i-1}}{\phi_{i+2}-\phi_{i+1}-\phi_{i}+\phi_{i-1}}-\frac{1}{\gamma}
$$

It can be checked that the value of $\alpha^{(e)}$ given by Eq. (155) coincides in this case with the analytical expression typically used in practice. For this purpose let us substitute into Eq. (155) the general numerical solution for this case given by

$$
\phi_{i}=A+B\left[\frac{1+\gamma(\alpha+1)}{1+\gamma(\alpha-1)}\right]^{i}
$$

where $A$ and $B$ are appropriate constants. After same simple algebra we obtain

$$
\alpha^{(e)}=1-\frac{1}{\gamma}
$$

which coincides with the standard critical value [3].

Eq. (155) can be used for deriving an iterative scheme for computation of $\alpha^{(e)}$ as follows:

(i) Solve the stabilized problem (23) by the FEM with an initial guess of $\alpha^{(e)}={ }^{0} \alpha^{(e)}$.

(ii) Compute an enhanced value ${ }^{\mathrm{i}} \bar{\alpha}^{(e)}$ by Eq. (155).

(iii) Repeat the numerical solution of the stabilized problem with a new value of $\alpha$ given by ${ }^{1} \alpha^{(e)}=$ $\frac{1}{2}\left({ }^{0} \alpha^{(e)}+{ }^{\mathrm{i}} \bar{\alpha}^{(e)}\right)$.

(iv) Repeat the process until a satisfactory stable numerical solution is found or else $\left\|^{i+1} \alpha^{(e)}-{ }^{i} \alpha^{(e)}\right\| \leqslant \varepsilon$, where $\varepsilon$ is a prescribed tolerance.

The computation of the term involving differences of the $\phi_{i}$ values in the r.h.s. of Eq. (155) can be difficult due to round off errors in zones where changes in the numerical solution are small. This problem can be overcome by the following procedure:

(i) If $\phi_{i+2}-\phi_{0} \leqslant \varepsilon$, where $\phi_{0}$ is prescribed value at the Dirichlet boundary and $\varepsilon$ is a very small number, then the first term of the r.h.s. of Eq. (155) is made equal to the unity. This correction eliminates the possible oscillations of the computed value of $\alpha^{(e)}$ in zones where the relative changes of the solution are small. In the examples shown next $\varepsilon=10^{-10}$ has been taken.

(ii) If ${ }^{i} \alpha^{(e)}>1$ or ${ }^{i} \alpha^{(e)}<0$ then ${ }^{i} \alpha^{(e)}=1$ and ${ }^{i} \alpha^{(e)}=0$ are, respectively, taken.

Figs. 9-11 show practical applications of this iterative process. In the first case the solution of the 1D advective-diffusive problem is attempted for $\gamma=5$ using a mesh of twenty linear elements. An initial value of ${ }^{0} \alpha^{(e)}=0.5$ is chosen for all elements. Fig. 9 shows the convergence of the solution for $\alpha^{(e)}$. Note that the critical value $\alpha^{(e)}=0.8$ is obtained in all elements after a few iterations. Fig. 9 also displays the convergence of the numerical solution for $\phi$ showing convergence to the 'exact' solution after three iterations.

Results for the same problem for $\gamma=25$ are shown in Fig. 10. Note that an excellent solution is obtained with just two iterations in this case. Seven iterations are however needed to obtain the critical value of $\alpha^{(e)}$ for all elements. Indeed in both cases it suffices to obtain a good approximation for $\alpha^{(e)}$ in the vicinity of the exit node and this always occurs after 2-3 iterations. 

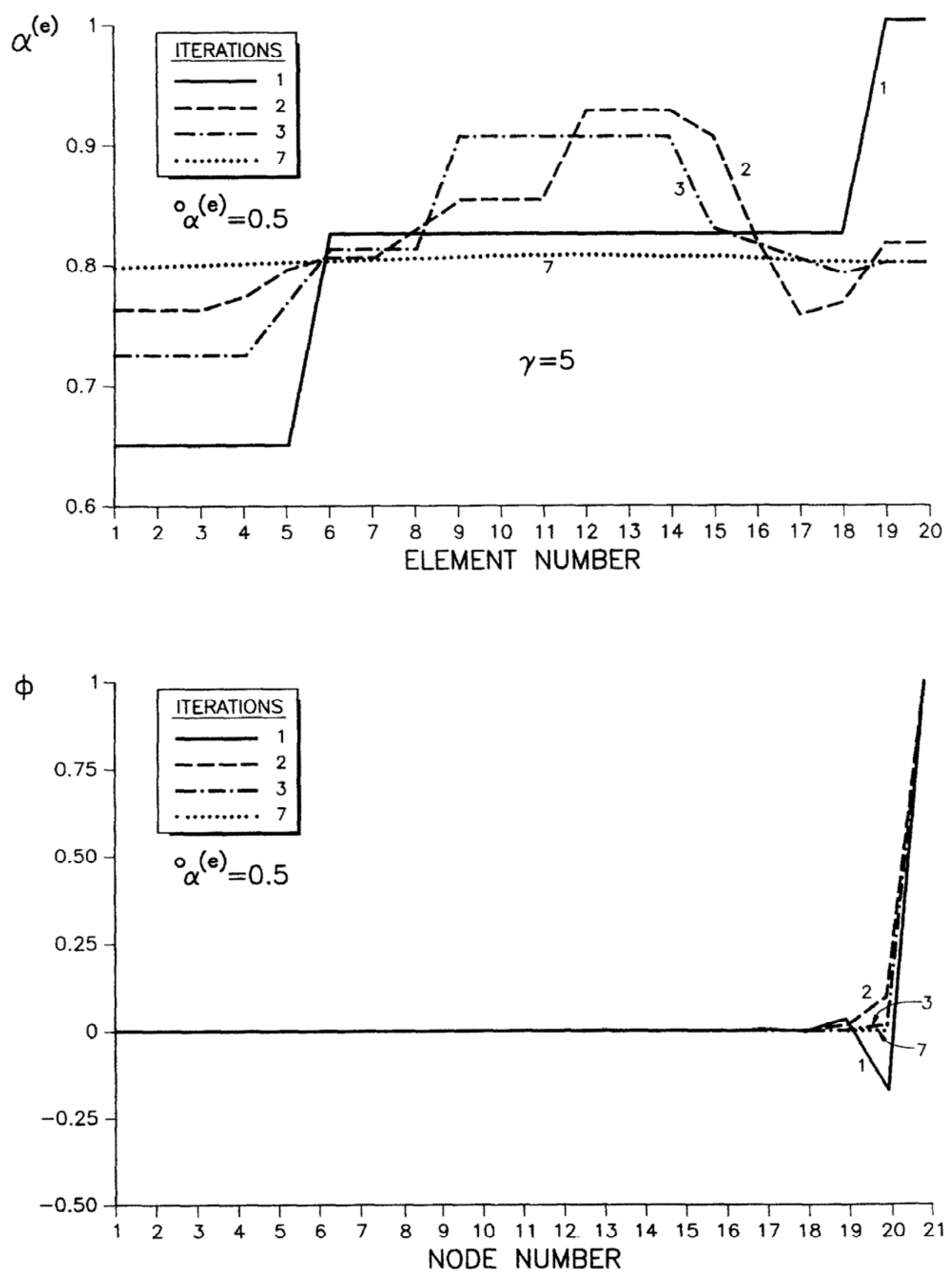

Fig. 9. One-dimensional advection-diffusion problem. $Q=0, \phi_{0}=0, \phi_{1}=1$. Convergence of the critical value of the element stabilization parameter $\alpha^{(e)}$ and of the numerical solution $\phi$. Solution obtained with 20 two node linear elements. Peclet number $\gamma=5 ;{ }^{0} \alpha^{(\varepsilon)}=0.5$. 

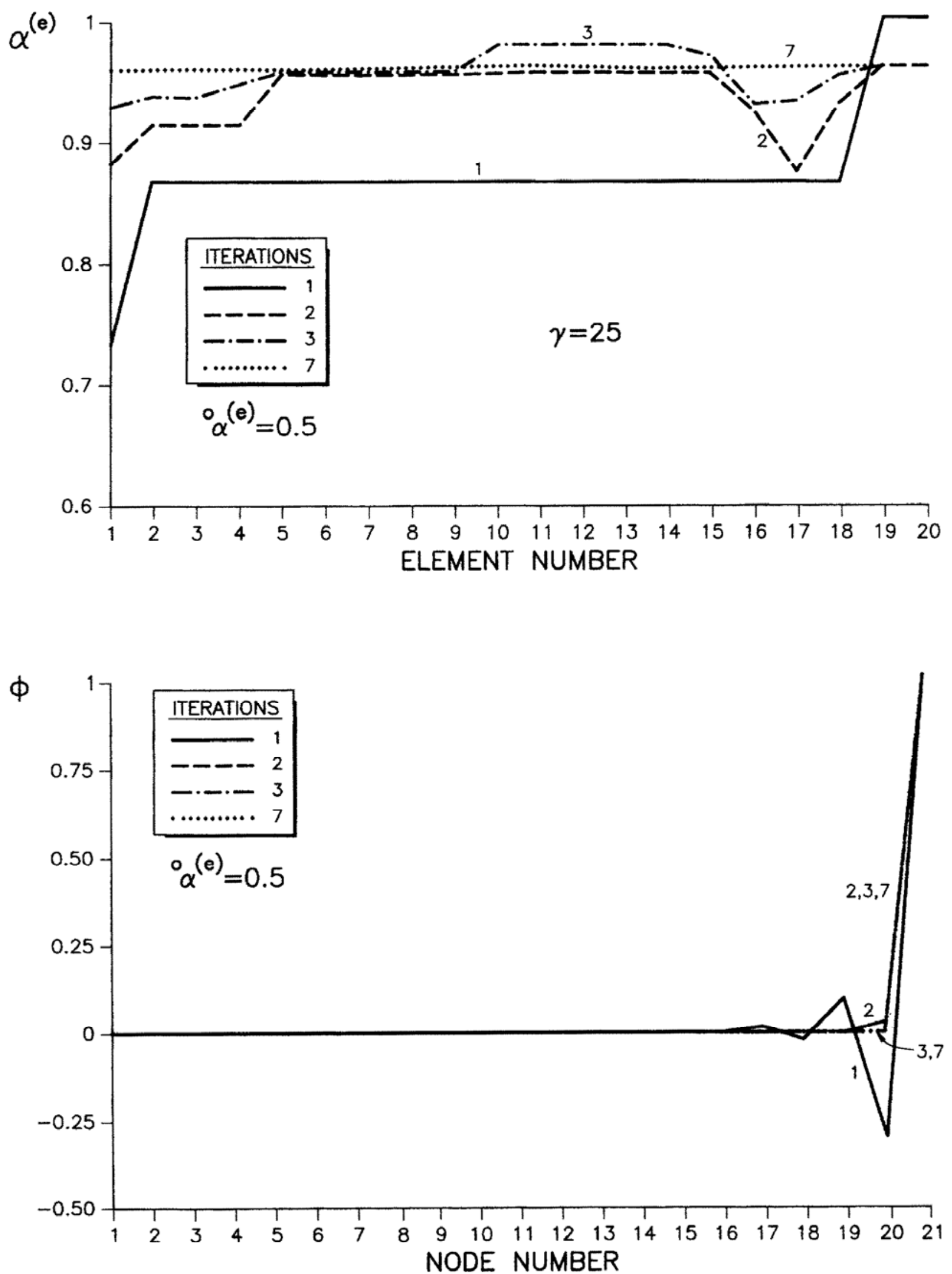

Fig. 10. One-dimensional advection-diffusion problem. $Q=0, \phi_{0}=0, \phi_{l}=1$. Convergence of the critical value of the element stabilization parameter $\alpha^{(e)}$ and of the numerical solution $\phi$. Solution obtained with 20 two node linear elements. Peclet number $\gamma=25 ;{ }^{\circ} \alpha^{(e)}=0.5$. 

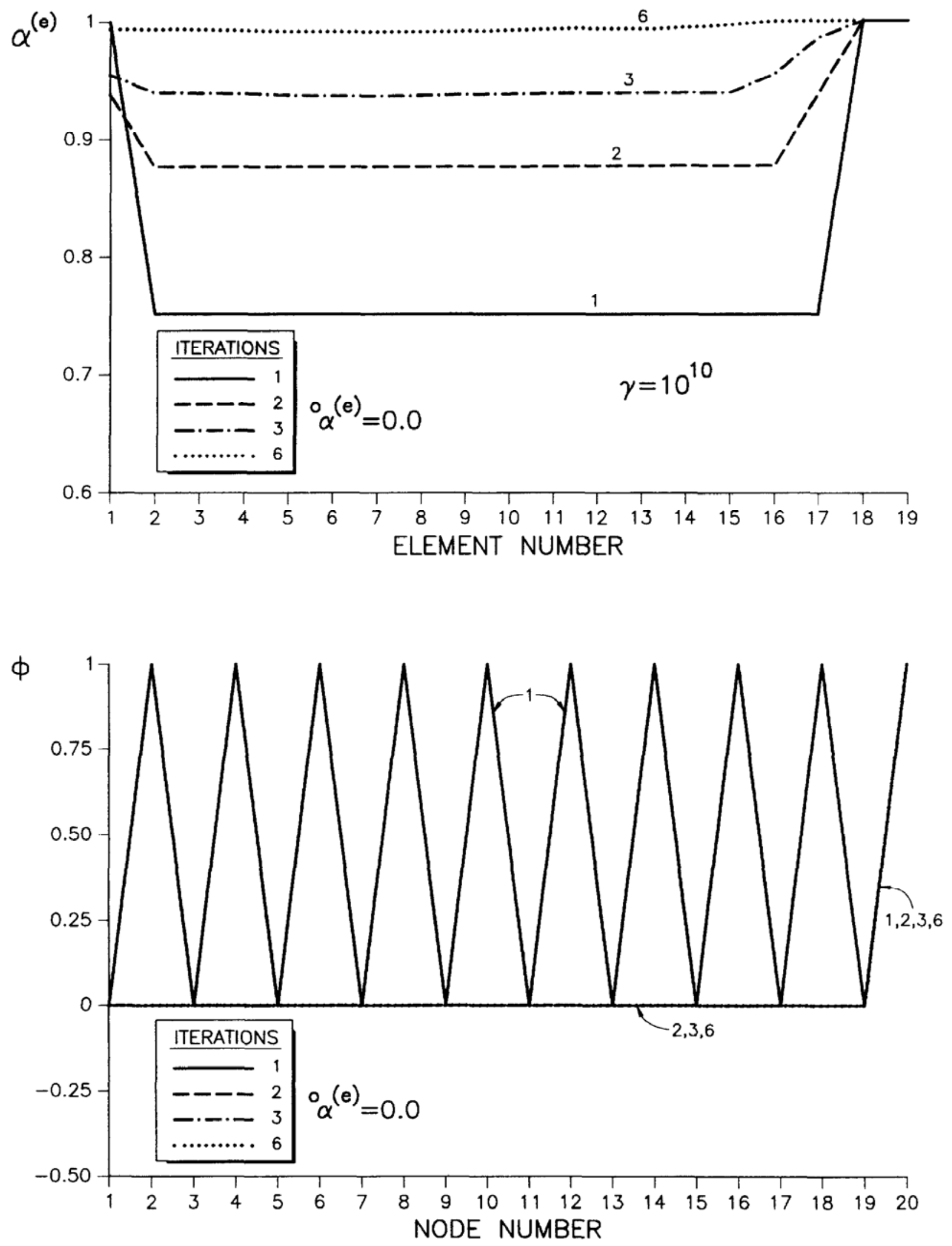

Fig. 11. One-dimensional advection-diffusion problem. $Q=0, \phi_{0}=0, \phi_{1}=1$. Convergence of the critical value of the element stabilization parameter $\alpha^{(e)}$ and of the numerical solution $\phi$. Solution obtained with 20 two node linear elements. Peclet number $\gamma=10^{10}:{ }^{0} \alpha^{(\epsilon)}=0.0$. 
Fig. 11 shows the solution of the same problem for $\gamma=10^{10}$ obtained now with an initial value of ${ }^{0} \alpha^{(e)}=0$ for all elements. Excellent results are again obtained after 2-3 iterations.

The possibilities of exploiting the form (148) for the numerical computation of the critical stabilization parameter are discussed more extensively in [28].

\section{Concluding remarks}

This paper has shown that the origins of many numerical stabilization methods for advective-diffusive transport and fluid flow problems lay in the higher-order governing differential equations incorporating the effect of the finite balance domain size $h$. Indeed standard conservation statements and the corresponding PDEs are recovered for the limit $h=0$. The finite increment form of the differential equations can be used as starting point to readily derive stabilized differences schemes via FD and FP approximations. Alternatively all well-known stabilized integral forms typically used in FV and FE practice emerge also as a consequence of the new stabilized governing differential equations. Extensions of the method to include the effect of reaction terms seem straightforward.

An interesting spin off result of this Finite Increment Calculus approach is a procedure for computing numerically the value of the critical stabilization parameter. This seems to offer a promising trend to obtain consistent values of the no longer mysterious stabilization parameter, therefore eliminating the need for heuristic predictions.

\section{Acknowledgments}

The author thanks the help of Mr. J. García in the computation of the numerical example shown in last section. Thanks are also given to Drs. M. Manzan, M. Goldschmit and R. Codina and Profs. S. Idelsohn, O.C. Zienkiewicz, R.L. Taylor and E.N. Dvorkin for many useful discussions. The name Finite Increment Calculus mentioned in the last section was suggested by Prof. O.C. Zienkiewicz.

\section{References}

[1] C. Hirsch, Numerical Computations of Internal and External Flow, Vol. 2 (John Wiley, 1990).

[2] S. Idelsohn and E. Oñate. Finite element and finite volumes. Two good friends, Int. J. Numer. Methods Engrg. 37 (1994) $3323-3341$.

[3] O.C. Zienkiewicz and R.L. Taylor, The Finite Element Method (McGraw Hill, Vol. I, 1989, Vol. II, 1991).

[4] A. Brooks and T.J.R. Hughes, Streamline upwind/Petrov-Galerkin formulation for convection dominated flows with particular emphasis on the incompressible Navier-Stokes equations, Comput. Methods Appl. Mech. Engrg. 32 (1982) 199-259.

[5] T.J.R. Hughes and M. Mallet, A new finite element formulations for computational fluid dynamics: III. The generalized streamline operator for multidimensional advective-diffusive systems, Comput. Methods Appl. Mech. Engrg. 58 (1986) 305-328.

[6] T.J.R. Hughes, G. Hauke and K. Jansen, Stabilized finite element methods in fluids: Inspirations, origins, status and recent developments, in: T.J.R. Hughes, E. Oñate and O.C. Zienkiewicz, eds., Recent Developments in Finite Element Analysis. A Book Dedicated to Robert L. Taylor (International Center for Numerical Methods in Engineering, Barcelona, Spain, 1994) $272-292$.

[7] R. Codina, A finite element model for incompressible flow problems, Ph.D. Thesis. Univ. Politécnica de Catalunya, Barcelona, June 1992.

[8] J.C. Heinrich, P.S. Hayakorn and O.C. Zienkiewicz, An upwind finite element scheme for two dimensional convective transport equations, Int. J. Numer. Methods Engrg. 11 (1977) 131-143.

[9] D.W. Kelly, S. Nakazawa, O.C. Zienkiewicz and J.C. Heinrich, A note on upwind and anisotropic balancing dissipation in finite element approximation to convective diffusion problems, Int. J. Numer. Methods Engrg. 15 (1980) 1705-1711.

[10] L.P. Franca, S.L. Frey and T.J.R. Hughes, Stabilized finite element methods: I. Application to the advective-diffusive model, Comput. Methods Appl. Mech. Engrg 95 (1992) 253-276.

[11] L.P. Franca and S.L. Frey, Stabilized finite element methods: II. The incompressible Navier-Stokes equations, Comput. Methods Appl. Mech. Engrg. 99 (1992) 209-233.

[12] I. Harari and T.J.R. Hughes, Stabilized finite element methods for steady advection-diffusion with production, Comput. Methods Appl. Mech. Engrg. 115 (1994) 165-191.

[13] E. Oñate, S. Idelsohn, O.C. Zienkiewicz and R.L. Taylor, A finite point method in computational mechanics. Applications to convective transport and fluid flow, Int. J. Numer. Methods Engrg. 39 (1996) 3839-3866. 
[14] E. Oñate, S. Idelsohn, O.C. Zienkiewicz, R.L. Taylor and C. Sacco, A stabilized finite point method for fluid mechanics, Comput. Methods Appl. Mech. Engrg. 39 (1996) 315-346.

[15] T.J.R. Hughes, Multiscale phenomena: Greens functions, subgrid scale models, bubbles and the origins of stabilized methods. Comput. Methods Appl. Mech. Engrg. 127 (1995) 387-401.

[16] F. Brezzi, M.O. Bristeau, L.P. Franca, M. Mallet and G. Rogé, A relationship between stabilized finite element methods and the Galerkin method with bubble functions, Comput. Methods Appl. Mech. Engrg. 96 (1992) 117-129.

[17] F. Brezzi, D. Marini and A. Russo, Pseudo residual-free bubbles and stabilized methods. in: J. Periaux et al., eds., Comput. Methods Appl. Sciences '96 (J. Wiley, 1996).

[18] F. Brezzi, L.P. Franca, T.J.R. Hughes and A. Russo, $b=\int g$, Comput. Methods Appl. Mech. Engrg., to appear.

[19] J. Donea, T. Belytschko and P. Smolinski, A generalized Galerkin method for steady state convection-diffusion problems with applications to quadratic shape functions elements. Comput. Methods Appl. Mech. Engrg. 48 (1985) 25-43.

[20] M.B. Goldschmit and E.N. Dvorkin, On the solution of the steady-state convection-diffusion equation using quadratic elements. A generalized Galerkin technique also reliable with distorted meshes, Engrg. Comput. 11(6) (1994) 565-579.

[21] J. Donea, A Taylor-Galerkin method for convective transport problems, Int. J. Numer. Methods Engrg. 20 (1984) $101-119$.

[22] O.C. Zienkiewicz and R. Codina. A general algorithm for compressible and incompressible flow. Part I: The split characteristic based scheme, Int. J. Numer. Methods Fluids 20 (1995) 869-885.

[23] O.C. Zienkiewicz, K. Morgan, B.V.K. Satya Sai, R. Codina and M. Vázquez, A general algorithm for compressible and incompressible flow. Part II: Tests on the explicit form, Int. J. Numer. Methods Fluids 20(8-9) (1995) 886-913.

[24] T.J.R. Hughes. L.P. Franca and G.M. Hulbert, A new finite element formulation for computational fluid dynamics: VIII. The Galerkin/least-squares method for advective-diffusive equations. Comput. Methods Appl. Mech. Engrg. 73 (1989) 173-189.

[25] J.C. Heinrich, On quadratic elements in finite element solutions of steady state convection-diffusion equations, Int. J. Numer. Methods Engrg. 15 (1980) 1041-1052.

[26] R. Codina, E. Oñate and M. Cervera. The intrinsic time for the streamline upwind Petrov-Galerkin formulation using quadratic elements. Comput. Methods Appl. Mech. Engrg. 94 (1992) 239-262.

[27] O.C. Zienkiewicz and J.Z. Zhu, The supercorvergent patch recovery (SPR) and adaptive finite element refinement. Comput. Methods Appl. Mech. Engrg. 101 (1992) 207-224.

[28] E. Oñate, J. García and S. Idelsohn, A procedure for computing the stabilization parameter for advective-diffusive problems, Publication CIMNE No. 100, Barcelona, June 1996.

[29] R. Codina, Comparison of some finite element methods for solving the diffusion-convection-reaction equation. Publication CIMNE No. 101, Barcelona, 1996.

[30] E. Oñate, On the stabilization of numerical solution for advective-diffusive transport and fluid flow problems, Publication CIMNE No. 81, Barcelona, October 1996. 